\title{
The computation of pairwise stable networks
}

Citation for published version (APA):

Herings, P. J-J., \& Zhan, Y. (2021). The computation of pairwise stable networks. Maastricht University, Graduate School of Business and Economics. GSBE Research Memoranda No. 004 https://doi.org/10.26481/umagsb.2021004

Document status and date:

Published: 26/01/2021

DOI:

10.26481/umagsb.2021004

Document Version:

Publisher's PDF, also known as Version of record

\section{Please check the document version of this publication:}

- A submitted manuscript is the version of the article upon submission and before peer-review. There can be important differences between the submitted version and the official published version of record.

People interested in the research are advised to contact the author for the final version of the publication, or visit the DOI to the publisher's website.

- The final author version and the galley proof are versions of the publication after peer review.

- The final published version features the final layout of the paper including the volume, issue and page numbers.

Link to publication

\footnotetext{
General rights rights.

- You may freely distribute the URL identifying the publication in the public portal. please follow below link for the End User Agreement:

www.umlib.nl/taverne-license

Take down policy

If you believe that this document breaches copyright please contact us at:

repository@maastrichtuniversity.nl

providing details and we will investigate your claim.
}

Copyright and moral rights for the publications made accessible in the public portal are retained by the authors and/or other copyright owners and it is a condition of accessing publications that users recognise and abide by the legal requirements associated with these

- Users may download and print one copy of any publication from the public portal for the purpose of private study or research.

- You may not further distribute the material or use it for any profit-making activity or commercial gain

If the publication is distributed under the terms of Article $25 \mathrm{fa}$ of the Dutch Copyright Act, indicated by the "Taverne" license above, 


\section{Maastricht University}

P. Jean-Jacques Herings, Yang Zhan

The Computation of Pairwise Stable Networks

$\mathrm{RM} / 21 / 004$

ISSN: $2666-8807$

\section{GSBE}

Maastricht University School of Business and Economics

Graduate School of Business and Economics

P.O Box 616

NL-6200 MD Maastricht

The Netherlands 


\title{
The Computation of Pairwise Stable Networks
}

\author{
P. Jean-Jacques Herings* and Yang Zhan ${ }^{\dagger}$
}

\begin{abstract}
One of the most important stability concepts for network formation is pairwise stability. We develop a homotopy algorithm that is effective in computing pairwise stable networks for a generic network formation problem. To do so, we reformulate the concept of pairwise stability as a Nash equilibrium of a non-cooperative game played by the links in the network and adapt the linear tracing procedure for non-cooperative games to the network formation problem. As a by-product of our main result, we obtain that the number of pairwise stable networks is generically odd. We apply the algorithm to the connections model.
\end{abstract}

KEYWORDS: Pairwise stable network; Computation of equilibria; Tracing procedure

MAThematics SubJect Classification: 90B10; 90C33; 91A43; 91-08

*P.J.J. Herings, Department of Economics, Maastricht University, P.O. Box 616, 6200 MD, Maastricht, The Netherlands. E-mail: P.Herings@maastrichtuniversity.nl

${ }^{\dagger}$ Y. Zhan, Department of Management Science and Engineering, School of Management and Engineering, Nanjing University, Nanjing, China. E-mail: zhanyang@nju.edu.cn 


\section{Introduction}

Networks are at the forefront of research in economics, operations research, and computer science as powerful tools to model social and economic interactions. A network consists of a finite set of nodes, representing agents, and a set of links, representing relationships between these agents. This paper considers the situation where agents make strategic decisions regarding the formation of links.

In network formation theory, Jackson and Wolinsky (1996) introduced the widely used concept of pairwise stability, see in particular Bloch and Jackson (2006), Calvó-Armengol and İlkılıç (2009), and Miyauchi (2016). A network is pairwise stable if for any pair of agents that is connected neither of them benefits from deleting their link and for any pair of agents that is not connected at least one of them loses from creating the link. Compared with Nash equilibrium, pairwise stability has both cooperative and non-cooperative aspects: Link deletion is a unilateral decision, whereas it requires the consent of both agents to establish a link.

Most of the literature considers unweighted networks, where each pair of agents is either linked or not. Recently, Bich and Morhaim (2020) study a weighted version, where the strength of each link is measured by a continuous variable. Agents can unilaterally decide to decrease the link strength, whereas it requires the consent of both agents to increase it. Bich and Morhaim (2020) prove that pairwise stable networks exist if all agents have quasi-concave and continuous utility functions. This is an important contribution as in unweighted networks, strong conditions are needed to guarantee the existence of pairwise stable networks, see Jackson and Watts (2001), Chakrabarti and Gilles (2007), and Hellmann (2013).

Further progress in this research program can be made by developing methods to solve strategic network formation problems numerically. For unweighted networks where many agents have dominant strategies, Leung (2020) develops an algorithm which makes it possible to decompose the network of interest into smaller subnetworks. For the subnetworks, pairwise stable networks are found by exhaustive search. The algorithm of Leung (2020) exploits the sparse structure of the problem and has attractive complexity properties.

This paper is the first to develop an effective method to compute pairwise stable networks in the general case. We assume that agents have multi-linear payoff functions, which enables us to interpret the model of Bich and Morhaim (2020) as the mixed strategy version of the model of Jackson and Wolinsky (1996).

Apart from existence issues, pairwise stability also suffers from multiplicity problems. For given payoff functions, there can be many pairwise stable networks. It is therefore essential to pay attention to the way a pairwise stable network is selected. In non-cooperative game theory, multiplicity of Nash equilibrium is a real concern (von Stengel, 1999; McLennan, 2005). The linear tracing procedure of Harsanyi and Selten (1988) addresses the equilibrium selection problem by formulating a process that transforms agents' prior beliefs into equilibrium beliefs. For normal-form games, the Nash equilibrium selected by the linear tracing procedure can be computed by the homotopy algorithm of Herings and Peeters (2001). This work has been extended to stochastic games in Herings and Peeters (2004) and Li and Dang (2020). It is also 
closely related to the global Newton method as developed in Govindan and Wilson (2003) for normal-form games and Govindan and Wilson (2009) for stochastic games.

Our algorithm adapts the linear tracing procedure of Harsanyi and Selten (1988) to select a pairwise stable network. To do so, we reformulate the network formation problem as a non-cooperative game played by the links. Then, we apply the linear tracing procedure to this auxiliary game. The auxiliary game features robust non-differentiabilities and is therefore outside the class of normal-form games that has been considered in the literature so far. We show that for a generic network formation problem, there is a unique path that transforms arbitrary prior beliefs of agents to beliefs that are compatible with a pairwise stable network.

As a by-product of our convergence proof, we obtain the result that for a generic network formation problem the number of pairwise stable networks is odd.

As a final result, we show how a suitably chosen transformation of variables makes it possible to obtain the path in the auxiliary game as the solution to an everywhere differentiable homotopy. Our algorithm therefore belongs to the class of differentiable homotopy methods. These methods are both useful to compute equilibria in general equilibrium models, see Eaves and Schmedders (1999) for an overview, and to compute equilibria in various classes of noncooperative games, see Herings and Peeters (2010) for a survey. This paper is the first to apply homotopy algorithms to network formation problems, problems that have non-cooperative as well as cooperative features.

The paper is organized as follows. Section 2 describes the network formation problem and the concept of pairwise stability. Section 3 introduces the linear tracing procedure for networks problems, Section 4 establishes that it is feasible and generically well-defined, and Section 5 transforms it into an everywhere differentiable homotopy. Section 6 presents an example involving the connections model. Section 7 describes the numerical implementation for the connections model. Section 8 presents a short conclusion.

\section{Pairwise Stable Networks}

Let $N=\{1,2, \ldots, n\}$ be the finite set of agents. The network relations among them are represented by an undirected graph, where the nodes represent the agents and the links stand for pairwise relations. Let $L=\{(i, j) \in N \times N \mid i<j\}$ be the set of links. For ease of notation, we use $i j$ instead of $(i, j)$ to denote the link between nodes $i$ and $j$.

An unweighted network on $N$ corresponds to a binary vector $g \in\{0,1\}^{L}$. For each link $i j \in L, g_{i j}=1$ if $i$ and $j$ are connected and $g_{i j}=0$ otherwise. For every $i j \in L$, for every $s \in\{0,1\}, g^{\prime}=\left(s, g_{-i j}\right)$ denotes the network such that $g_{k \ell}^{\prime}=g_{k \ell}$ for every $k \ell \neq i j$ and $g_{i j}^{\prime}=s$. Let $G=\{0,1\}^{L}$ be the set of all possible unweighted networks and, for every $i j \in L$, let $G_{-i j}=\{0,1\}^{L \backslash\{i j\}}$ be the set of all possible unweighted networks without taking link $i j$ into consideration.

Agent $i \in N$ has a payoff function $v^{i}: G \rightarrow \mathbb{R}$. We denote the tuple of payoff functions of all agents by $v=\left(v^{i}\right)_{i \in N}$. 
In their seminal paper, Jackson and Wolinsky (1996) introduce the following notion of stability. ${ }^{1}$

Definition 1 (Strictly pairwise stable unweighted network). An unweighted network $g \in G$ is strictly pairwise stable with respect to $v$ if, for every $i j \in L$,

1. if $g_{i j}=1$, then $v^{i}\left(0, g_{-i j}\right) \leq v^{i}(g)$ and $v^{j}\left(0, g_{-i j}\right) \leq v^{j}(g)$,

2. if $g_{i j}=0$, then $v^{i}\left(1, g_{-i j}\right)>v^{i}(g)$ implies $v^{j}\left(1, g_{-i j}\right)<v^{j}(g)$.

Strict pairwise stability incorporates that link deletion is a unilateral decision, whereas it requires the consent of both agents to establish a link. Moreover, to form a link, it is sufficient that one agent strictly benefits from doing so, whereas the other agent might be indifferent. This is somewhat different from the usual assumption in cooperative game theory, where for a coalition to deviate, all its members should strictly benefit. In Section 5 of Jackson and Wolinsky (1996), the authors also discuss a weaker notion of stability, which we call pairwise stability. This notion requires that if $g_{i j}=0$ for some link $i j \in L$, then $v^{i}\left(1, g_{-i j}\right)>v^{i}(g)$ implies $v^{j}\left(1, g_{-i j}\right) \leq v^{j}(g)$.

In this paper, we follow Bich and Morhaim (2020) and go beyond unweighted networks. We represent each link $i j \in L$ by a real number $x_{i j} \in[0,1]$. As argued in Bich and Morhaim (2020), it is natural to quantify the weight of a relationship in a continuous way. The weight can measure intensity, level of confidence, geographical distance, and so on. In this paper, we interpret $x_{i j}$ as the probability that link $i j$ forms.

Let $\Delta(G)=[0,1]^{L}$ be the set of all possible networks and, for every ij $\in L$, let $\Delta\left(G_{-i j}\right)=$ $[0,1]^{L \backslash\{i j\}}$ be the set of all possible networks without taking link $i j$ into consideration. Given $x \in \Delta(G)$, the expected payoff received by agent $i$ is

$$
u^{i}(x)=\sum_{g \in G} \pi_{g}(x) v^{i}(g)
$$

where the probability $\pi_{g}(x)$ that network $g$ forms is given by

$$
\pi_{g}(x)=\prod_{i j \in L}\left(x_{i j} g_{i j}+\left(1-x_{i j}\right)\left(1-g_{i j}\right)\right) .
$$

Similarly, for every $k \ell \in L$, for every $x_{-k \ell} \in \Delta\left(G_{-k \ell}\right)$, we define

$$
\pi_{g_{-k \ell}}\left(x_{-k \ell}\right)=\prod_{i j \in L \backslash\{k \ell\}}\left(g_{i j} x_{i j}+\left(1-g_{i j}\right)\left(1-x_{i j}\right)\right) .
$$

We denote the tuple of payoff functions of all agents by $u=\left(u^{i}\right)_{i \in N}$.

Bich and Morhaim (2020) extend the definition of pairwise stability for unweighted networks to networks in $\Delta(G)$ in the following way.

Definition 2 (Pairwise stable networks). A network $x \in \Delta(G)$ is pairwise stable with respect to $u$ if, for every $i j \in L$,

\footnotetext{
${ }^{1}$ In the terminology of Jackson and Wolinsky (1996), this is called a pairwise stable network.
} 
1. for every $s \in\left[0, x_{i j}\right), u^{i}\left(s, x_{-i j}\right) \leq u^{i}(x)$ and $u^{j}\left(s, x_{-i j}\right) \leq u^{j}(x)$,

2. for every $s \in\left(x_{i j}, 1\right], u^{i}\left(s, x_{-i j}\right)>u^{i}(x)$ implies $u^{j}\left(s, x_{-i j}\right) \leq u^{j}(x)$.

That is, $x$ is pairwise stable if there are no two agents that can gain from strengthening their connection and there is no single agent that can gain by weakening it. The idea is that a single agent sufficies to weaken a link, but consent of both agents is required in order to strengthen it.

When each component of $x$ is required to be equal to zero or one, the notion of pairwise stable network reverts to the one of pairwise stable unweighted network. ${ }^{2}$

We use a tuple $W=\left\langle N, \Delta(G),\left(u^{i}\right)_{i \in N}\right\rangle$ to represent a network formation problem involving the agents in $N$. The set of all network formation problems with set of agents $N$ is denoted by $\mathcal{W}$. For every $W \in \mathcal{W}$, we use $\operatorname{PS}(W)$ to denote the set of pairwise stable networks of $W$. Theorem 2 in Bich and Morhaim (2020) establishes that for every $W \in \mathcal{W}$, a pairwise stable network exists, so $\operatorname{PS}(W) \neq \emptyset$.

\section{The Linear Tracing Procedure for Networks}

In this section, we reformulate the concept of pairwise stability as a Nash equilibrium of a noncooperative game, where the agents in the game correspond to the links in the network. Next, we use the linear tracing procedure of Harsanyi and Selten (1988) to select a Nash equilibrium in this game, which therefore corresponds to selecting a pairwise stable network in the network formation problem.

Lemma 1 reformulates the inequalities in Definition 2.

Lemma 1. Let $W \in \mathcal{W}$. It holds that $x \in \operatorname{PS}(W)$ if and only if there exists $\alpha, \beta \in \mathbb{R}_{+}^{L}$ such that

$$
\begin{aligned}
\min \left\{\frac{\partial}{\partial x_{i j}} u^{i}(x), \frac{\partial}{\partial x_{i j}} u^{j}(x)\right\}+\alpha_{i j}-\beta_{i j} & =0, \quad i j \in L, \\
\alpha_{i j} x_{i j} & =0, \quad i j \in L, \\
\beta_{i j}\left(1-x_{i j}\right) & =0, \quad i j \in L .
\end{aligned}
$$

Proof. $(\Rightarrow)$

Let $x \in \operatorname{PS}(W)$.

For every $i j \in L$, it holds that $u^{i}\left(s, x_{-i j}\right)$ is affine in $s \in[0,1]$, so $\frac{\partial}{\partial x_{i j}} u^{i}(x)$ is well-defined. Condition 1 of Definition 2 implies that, for every $i j \in L$ such that $x_{i j}>0, \frac{\partial}{\partial x_{i j}} u^{i}(x) \geq 0$ and $\frac{\partial}{\partial x_{i j}} u^{j}(x) \geq 0$, so $\min \left\{\frac{\partial}{\partial x_{i j}} u^{i}(x), \frac{\partial}{\partial x_{i j}} u^{j}(x)\right\} \geq 0$. Condition 2 of Definition 2 implies that, for every $i j \in L$ such that $x_{i j}<1, \min \left\{\frac{\partial}{\partial x_{i j}} u^{i}(x), \frac{\partial}{\partial x_{i j}} u^{j}(x)\right\} \leq 0$.

Let $i j \in L$. If $x_{i j}=0$, then the previous paragraph implies $\min \left\{\frac{\partial}{\partial x_{i j}} u^{i}(x), \frac{\partial}{\partial x_{i j}} u^{j}(x)\right\} \leq 0$. We define $\alpha_{i j}=-\min \left\{\frac{\partial}{\partial x_{i j}} u^{i}(x), \frac{\partial}{\partial x_{i j}} u^{j}(x)\right\}$ and $\beta_{i j}=0$. If $x_{i j} \in(0,1)$, then the previous paragraph implies $\min \left\{\frac{\partial}{\partial x_{i j}} u^{i}(x), \frac{\partial}{\partial x_{i j}} u^{j}(x)\right\}=0$. We define $\alpha_{i j}=\beta_{i j}=0$. If $x_{i j}=1$,

\footnotetext{
${ }^{2}$ As observed in endnote 14 of Bich and Morhaim (2020), this equivalence does not hold when instead of being linear, utilities are only required to be quasi-concave in $x_{i j}$.
} 
then the previous paragraph implies $\min \left\{\frac{\partial}{\partial x_{i j}} u^{i}(x), \frac{\partial}{\partial x_{i j}} u^{j}(x)\right\} \geq 0$. We define $\alpha_{i j}=0$ and $\beta_{i j}=\min \left\{\frac{\partial}{\partial x_{i j}} u^{i}(x), \frac{\partial}{\partial x_{i j}} u^{j}(x)\right\}$.

It is easily verified that $(x, \alpha, \beta) \in[0,1]^{L} \times \mathbb{R}_{+}^{L} \times \mathbb{R}_{+}^{L}$ yields a solution to (2)-(4).

$(\Leftarrow)$

Let $(x, \alpha, \beta) \in[0,1]^{L} \times \mathbb{R}_{+}^{L} \times \mathbb{R}_{+}^{L}$ be a solution to (2)-(4).

Let $i j \in L$. If $\min \left\{\frac{\partial}{\partial x_{i j}} u^{i}(x), \frac{\partial}{\partial x_{i j}} u^{j}(x)\right\}<0$, then $\alpha_{i j}, \beta_{i j} \geq 0$ and (2) imply $\alpha_{i j}>0$, so $x_{i j}=0$ by (3), and next $\beta_{i j}=0$ by (4). If $\min \left\{\frac{\partial}{\partial x_{i j}} u^{i}(x), \frac{\partial}{\partial x_{i j}} u^{j}(x)\right\}>0$, then $\alpha_{i j}, \beta_{i j} \geq 0$ and (2) imply $\beta_{i j}>0$, so $x_{i j}=1$ by (4), and $\alpha_{i j}=0$ by (3). We conclude that

$$
x_{i j}= \begin{cases}0, & \text { if } \min \left\{\frac{\partial}{\partial x_{i j}} u^{i}(x), \frac{\partial}{\partial x_{i j}} u^{j}(x)\right\}<0, \\ 1, & \text { if } \min \left\{\frac{\partial}{\partial x_{i j}} u^{i}(x), \frac{\partial}{\partial x_{i j}} u^{j}(x)\right\}>0 .\end{cases}
$$

Since $u^{i}$ and $u^{j}$ are multi-linear functions, we have, for every $s \in[0,1]$,

$$
\begin{gathered}
u^{i}\left(s, x_{-i j}\right)=u^{i}(x)+\left(s-x_{i j}\right) \frac{\partial}{\partial x_{i j}} u^{i}(x), \\
u^{j}\left(s, x_{-i j}\right)=u^{j}(x)+\left(s-x_{i j}\right) \frac{\partial}{\partial x_{i j}} u^{j}(x) .
\end{gathered}
$$

We now verify Condition 1 of Definition 2. Let $s \in\left[0, x_{i j}\right)$. It follows that $x_{i j}>0$, so, by $(5)$,

$$
\frac{\partial}{\partial x_{i j}} u^{i}(x) \geq \min \left\{\frac{\partial}{\partial x_{i j}} u^{i}(x), \frac{\partial}{\partial x_{i j}} u^{j}(x)\right\} \geq 0 .
$$

By (6), we have that $u^{i}\left(s, x_{-i j}\right) \leq u^{i}(x)$. The same argument shows that $u^{j}\left(s, x_{-i j}\right) \leq u^{j}(x)$.

We next check Condition 2 of Definition 2. Let $s \in\left(x_{i j}, 1\right]$. It follows that $x_{i j}<1$, so, by (5), $\min \left\{\frac{\partial}{\partial x_{i j}} u^{i}(x), \frac{\partial}{\partial x_{i j}} u^{j}(x)\right\} \leq 0$. By (6), we have that $u^{i}\left(s, x_{-i j}\right) \leq u^{i}(x)$ or $u^{j}\left(s, x_{-i j}\right) \leq$ $u^{j}(x)$. Now $u^{i}\left(s, x_{-i j}\right)>u^{i}(x)$ implies $u^{j}\left(s, x_{-i j}\right) \leq u^{j}(x)$ as desired.

Since Conditions 1 and 2 of Definition 2 hold, it follows that $x$ is a pairwise stable network.

We now derive a closed-form solution for the partial derivatives of the utility function. For every $i j \in L$, let $f_{i j}^{i}:[0,1]^{L \backslash\{i j\}} \rightarrow \mathbb{R}$ denote the partial derivative of $u^{i}$ with respect to $x_{i j}$, that is,

$$
f_{i j}^{i}\left(x_{-i j}\right)=\frac{\partial}{\partial x_{i j}} u^{i}\left(s, x_{-i j}\right)=\sum_{g \in G}\left(2 g_{i j}-1\right) \pi_{g_{-i j}}\left(x_{-i j}\right) v^{i}(g),
$$

where the choice of $s \in[0,1]$ is irrelevant since $u^{i}\left(s, x_{-i j}\right)$ is affine in $s$. Note that the coefficient $2 g_{i j}-1$ is equal to 1 if $g_{i j}=1$ and equal to -1 if $g_{i j}=0$. The function $f_{i j}^{j}:[0,1]^{L \backslash\{i j\}} \rightarrow \mathbb{R}$ is defined analogously. Let $f_{i j}:[0,1]^{L \backslash\{i j\}} \rightarrow \mathbb{R}$ be defined as the minimum of $f_{i j}^{i}$ and $f_{i j}^{j}$, so

$$
f_{i j}\left(x_{-i j}\right)=\min \left\{f_{i j}^{i}\left(x_{-i j}\right), f_{i j}^{j}\left(x_{-i j}\right)\right\}, \quad x_{-i j} \in[0,1]^{L \backslash\{i j\}} .
$$

Conditions (2)-(4) of Lemma 1 correspond to the first-order condition of the problem

$$
x_{i j} \in \arg \max _{s \in[0,1]} s f_{i j}\left(x_{-i j}\right), \quad i j \in L .
$$

Therefore, the problem of finding a pairwise stable network of $W$ is equivalent to the problem of finding a Nash equilibrium of the game with set of agents $L$, each agent ij $\in L$ having 
two pure strategies, corresponding to forming a link or not, and payoff functions given by the objective in (8). We denote this game by $\Gamma(W)=\left\langle L,\left(f_{i j}\right)_{i j \in L}\right\rangle$. The next corollary summarizes our findings so far.

Corollary 1. Let $W \in \mathcal{W}$. It holds that $x \in \operatorname{PS}(W)$ if and only if $x$ is a Nash equilibrium of the game $\Gamma(W)$.

The equivalence between pairwise stability and Nash equilibrium motivates us to exploit methods used in non-cooperative game theory for computing and selecting Nash equilibria and develop an effective approach for finding pairwise stable networks. More precisely, we adapt the linear tracing procedure of Harsanyi and Selten (1988) to select a pairwise stable network in a network formation problem. Harsanyi and Selten (1988) consider finite games and Herings and Peeters (2004) extend the linear tracing procedure to finite stochastic games. The game $\Gamma(W)$ belongs to neither of these classes. The non-differentiabilities caused by the minimum operator in the definition of $f_{i j}$ poses new challenges that have to be taken care of.

The linear tracing procedure models a process by which agents update their beliefs. When formulated in terms of our network formation problem, the agents start out from common prior beliefs $p \in \Delta(G)$ and optimize against this common prior, leading to best responses $x \in \Delta(G)$. If $x$ happens to be equal to $p$, then the initial beliefs are confirmed, the prior beliefs constitute a Nash equilibrium, and the process of updating beliefs stops. But usually, $x \neq p$ and agents update their prior beliefs by giving a weight $1-t$ to the initial prior beliefs and $t$ to the best response $x$, for $t$ close to 0 . This leads to a new prior belief $(1-t) p+t x$ against which optimization takes place. This process of belief adaptation continues until a Nash equilibrium is found.

More formally, for every $t \in[0,1]$, let $W^{t}$ be the network formation problem such that with probability $t$ the agents have utility functions as in $W$ and with probability $1-t$ they are playing against the prior $p \in \Delta(G)$. The expected payoff of forming link $i j \in L$ with probability $x_{i j}$ is then given by

$$
t u^{i}(x)+(1-t) u^{i}\left(x_{i j}, p_{-i j}\right) .
$$

At $t=0$, this expression equals $u^{i}\left(x_{i j}, p_{-i j}\right)$. That is, when determining $x_{i j}$, agent $i$ assumes that the probabilities in the rest of the network are given by $p_{-i j}$. It is clear that $W^{0}$ is a trivial network formation problem without strategic interaction. The problem $W^{1}$ coincides with the original network formation problem $W$.

The linear tracing procedure is designed such that it links a pairwise stable network of $W^{0}$ to a pairwise stable network of $W^{1}$. The set of all pairwise stable networks related to $W^{t}$, $t \in[0,1]$, is denoted by

$$
\mathscr{L}(W, p)=\left\{(t, x) \in[0,1] \times \Delta(G) \mid x \in \operatorname{PS}\left(W^{t}\right)\right\} .
$$

We can apply Lemma 1 to $W^{t}$. For every $i j \in L$, let $F_{i j}^{i}:[0,1] \times[0,1]^{L \backslash\{i j\}} \rightarrow \mathbb{R}$ denote the partial derivative of $t u^{i}(x)+(1-t) u^{i}\left(x_{i j}, p_{-i j}\right)$ with respect to $x_{i j}$, that is

$$
F_{i j}^{i}\left(t, x_{-i j}\right)=t f_{i j}^{i}\left(x_{-i j}\right)+(1-t) f_{i j}^{i}\left(p_{-i j}\right) .
$$


The function $F_{i j}^{j}:[0,1] \times[0,1]^{L \backslash\{i j\}} \rightarrow \mathbb{R}$ is defined analogously. Let $F_{i j}:[0,1] \times$ $[0,1]^{L \backslash\{i j\}} \rightarrow \mathbb{R}$ be defined by

$$
F_{i j}\left(t, x_{-i j}\right)=\min \left\{F_{i j}^{i}\left(t, x_{-i j}\right), F_{i j}^{j}\left(t, x_{-i j}\right)\right\}, \quad\left(t, x_{-i j}\right) \in[0,1] \times[0,1]^{L \backslash\{i j\}} .
$$

We immediately obtain the following corollary.

Corollary 2. Let $W \in \mathcal{W}$. For every $t \in[0,1]$, it holds that $x \in \operatorname{PS}\left(W^{t}\right)$ if and only if there exists $\alpha, \beta \in \mathbb{R}_{+}^{L}$ such that

$$
\begin{aligned}
F_{i j}\left(t, x_{-i j}\right)+\alpha_{i j}-\beta_{i j} & =0, \quad i j \in L, \\
\alpha_{i j} x_{i j} & =0, \quad i j \in L, \\
\beta_{i j}\left(1-x_{i j}\right) & =0, \quad i j \in L,
\end{aligned}
$$

if and only if

$$
x_{i j} \in \arg \max _{s \in[0,1]} s F_{i j}\left(t, x_{-i j}\right), \quad i j \in L,
$$

if and only if $x$ is a Nash equilibrium of the game $\Gamma\left(W^{t}\right)=\left\langle L,\left(F_{i j}(t, \cdot)\right)_{i j \in L}\right\rangle$.

\section{The Structure of the Linear Tracing Procedure}

The linear tracing procedure is said to be feasible if there exists a path in $\mathscr{L}(W, p)$ connecting a pairwise stable network of $W^{0}$ to a pairwise stable network of $W^{1}$. It is said to be well-defined if the path is uniquely determined, which implies that a unique pairwise stable network of $W$ is selected.

The theorem below claims that the linear tracing procedure is feasible for all network formation problems.

Theorem 1. For every $W \in \mathcal{W}$, the linear tracing procedure is feasible.

Proof. Let $W \in \mathcal{W}$ and $t \in[0,1]$. By Corollary 2 it holds that $x \in \operatorname{PS}\left(W^{t}\right)$ if and only if

$$
x_{i j} \in \arg \max _{s \in[0,1]} s F_{i j}\left(t, x_{-i j}\right), \quad i j \in L .
$$

We define the correspondence $\varphi:[0,1] \times \Delta(G) \rightarrow \Delta(G)$ by

$$
\varphi(t, x)=\left\{\hat{x} \in \Delta(G) \mid \text { for every } i j \in L, \hat{x}_{i j} \in \arg \max _{s \in[0,1]} s F_{i j}\left(t, x_{-i j}\right)\right\},
$$

so $x \in \varphi(t, x)$ if and only if $(t, x) \in \mathscr{L}(W, p)$. The well-known maximum theorem implies that $\varphi$ is upper hemi-continuous. It is clear that $\Delta(G)$ is a non-empty, compact, and convex set. From Mas-Colell's fixed point theorem (Mas-Colell, 1974), the set of fixed points $T=$ $\{(t, x) \in[0,1] \times \Delta(G) \mid x \in \varphi(t, x)\}$ contains a component $T^{\mathrm{c}}$ such that $(\{0\} \times \Delta(G)) \cap T^{\mathrm{c}} \neq$ $\emptyset$ and $(\{1\} \times \Delta(G)) \cap T^{\mathrm{c}} \neq \emptyset$.

Since $\mathscr{L}(W, p)$ is a set that can be described by a finite number of polynomial inequalities, it is a semi-algebraic set and all its components are also path-connected, so any two points in a component can be joined by a path, see Schanuel, Simon, and Zame (1991). It follows that the linear tracing procedure is feasible. 
We show next that for a typical network formation problem, the linear tracing procedure is well-defined. To make this statement rigorous, we decompose $\mathscr{L}(W, p)$ into differentiable pieces. Non-differentiabilities occur when $x_{i j}$ changes along the path from 0 to a positive probability or vice versa and when $1-x_{i j}$ changes from 0 to a positive probability or vice versa. For every $i j \in L$, define the set $S_{i j}=\left\{s_{i j}^{0}, s_{i j}^{1}\right\}$. The meaning of $s_{i j}^{0}$ is that the best choice is not to form link $i j$, whereas $s_{i j}^{1}$ indicates that it is optimal to create link $i j$. Let $S=\cup_{i j \in L} S_{i j}$. A set $B \subset S$ is called admissible if, for every $i j \in L, B \cap S_{i j} \neq \emptyset$. The collection of all admissible subsets $B$ of $S$ is denoted by $\mathcal{B}$.

An additional non-differentiability occurs because the payoff function $F_{i j}=\min \left\{F_{i j}^{i}, F_{i j}^{j}\right\}$ is non-differentiable. Let $C: L \rightarrow N$ be a function such that, for every $i j \in L, C(i j) \in\{i, j\}$. The function $C$ chooses for each link $i j \in L$ an agent $C(i j)$ such that the minimal value of $F_{i j}$ is attained by $F_{i j}^{C(i j)}$. The collection of all such functions $C$ is denoted by $\mathcal{C}$.

We use pairs $(B, C) \in \mathcal{B} \times \mathcal{C}$ to decompose $\mathscr{L}(W, p)$ into subsets $\mathscr{L}(W, p, B, C)$ that are differentiable manifolds with boundary. Formally, $\mathscr{L}(W, p, B, C)$ is given by

$$
\mathscr{L}(W, p, B, C)=\left\{(t, x) \in \mathscr{L}(W, p) \mid \begin{array}{rl} 
& \forall i j \in L, \\
& C(i j)=i \Rightarrow F_{i j}^{i}\left(t, x_{-i j}\right) \leq F_{i j}^{j}\left(t, x_{-i j}\right), \\
& C(i j)=j \Rightarrow F_{i j}^{j}\left(t, x_{-i j}\right) \leq F_{i j}^{i}\left(t, x_{-i j}\right), \\
& s_{i j}^{1} \notin B \Rightarrow x_{i j}=0, \\
& s_{i j}^{0} \notin B \Rightarrow x_{i j}=1, \\
& s_{i j}^{1} \in B \Rightarrow F_{i j}\left(t, x_{-i j}\right) \geq 0, \\
& \left.s_{i j}^{0} \in B \Rightarrow F_{i j}\left(t, x_{-i j}\right) \leq 0\right\} .
\end{array}\right.
$$

It follows that

$$
\mathscr{L}(W, p)=\cup_{(B, C) \in \mathcal{B} \times \mathcal{C}} \mathscr{L}(W, p, B, C) .
$$

For every $(B, C) \in \mathcal{B} \times \mathcal{C}$, we define $\mathscr{O}(W, p, B, C)$ as the set of solutions $(t, x, \alpha, \beta) \in$ $\mathbb{R} \times \mathbb{R}^{L} \times \mathbb{R}^{L} \times \mathbb{R}^{L}$ to the following system of equalities and inequalities.

$$
\begin{aligned}
F_{i j}^{i}\left(t, x_{-i j}\right)+\alpha_{i j}-\beta_{i j} & =0, \quad i j \in L, C(i j)=i, \\
F_{i j}^{j}\left(t, x_{-i j}\right)+\alpha_{i j}-\beta_{i j} & =0, \quad i j \in L, C(i j)=j, \\
x_{i j} & =0, \quad s_{i j}^{1} \notin B, \\
x_{i j}-1 & =0, \quad s_{i j}^{0} \notin B, \\
\beta_{i j} & =0, \quad s_{i j}^{0} \in B, \\
\alpha_{i j} & =0, \quad s_{i j}^{1} \in B, \\
F_{i j}^{j}\left(t, x_{-i j}\right)-F_{i j}^{i}\left(t, x_{-i j}\right) & \geq 0, \quad C(i j)=i, \\
F_{i j}^{i}\left(t, x_{-i j}\right)-F_{i j}^{j}\left(t, x_{-i j}\right) & \geq 0, \quad C(i j)=j, \\
1-x_{i j} & \geq 0, \quad s_{i j}^{0} \in B, \\
x_{i j} & \geq 0, \quad s_{i j}^{1} \in B, \\
\alpha_{i j} & \geq 0, \quad s_{i j}^{1} \notin B, \\
\beta_{i j} & \geq 0, \quad s_{i j}^{0} \notin B, \\
t & \geq 0, \\
-t+1 & \geq 0 .
\end{aligned}
$$


Theorem 2. Let $(W, p) \in \mathcal{W} \times \Delta(G)$. For every $(B, C) \in \mathcal{B} \times \mathcal{C}$, the sets $\mathscr{L}(W, p, B, C)$ and $\mathscr{O}(W, p, B, C)$ are $C^{\infty}$ diffeomorphic.

Proof. Let $(B, C) \in \mathcal{B} \times \mathcal{C}$. Define the function $\varphi:[0,1] \times \Delta(G) \rightarrow \mathbb{R} \times \mathbb{R}^{L} \times \mathbb{R}^{L} \times \mathbb{R}^{L}$ by $\varphi(t, x)=(t, x, \alpha, \beta)$, where, for every $i j \in L, \alpha_{i j}=0$ if $s_{i j}^{1} \in B, \alpha_{i j}=-F_{i j}^{C(i j)}\left(t, x_{-i j}\right)$ if $s_{i j}^{1} \notin B, \beta_{i j}=0$ if $s_{i j}^{0} \in B$, and $\beta_{i j}=F_{i j}^{C(i j)}\left(t, x_{-i j}\right)$ if $s_{i j}^{0} \notin B$. It holds that $\varphi$ is a $C^{\infty}$ diffeomorphism such that $\varphi(t, x) \in \mathscr{O}(W, p, B, C)$ if and only if $(t, x) \in \mathscr{L}(W, p, B, C)$.

From Theorem 2 it follows that the structural properties of $\mathscr{L}(W, p, B, C)$ coincide with those of $\mathscr{O}(W, p, B, C)$.

A network formation problem $W \in \mathcal{W}$ is completely determined by the tuple of utility functions $v$, which can be represented by a vector in $\mathbb{R}^{n 2^{|L|}}$. The standard topology and measure on $\mathbb{R}^{n 2^{|L|}}$ therefore induce a topology and measure on network formation problems. The analysis of the system of equations and inequalities in (10)-(23) provides the following result.

Theorem 3. For an open set $(W, p) \in \mathcal{W} \times \Delta(G)$ with full Lebesgue measure, for every $(B, C) \in \mathcal{B} \times \mathcal{C}$, the set $\mathscr{L}(W, p, B, C)$ is a compact 1-dimensional $C^{\infty}$ manifold with boundary. A boundary point $(t, x)$ of $\mathscr{L}(W, p, B, C)$ is:

(i) Either a boundary point of exactly one $\mathscr{L}\left(W, p, B^{\prime}, C\right)$ with $B^{\prime} \neq B$. Moreover, $B$ and $B^{\prime}$ differ in exactly one element.

(ii) Or a boundary point of exactly one $\mathscr{L}\left(W, p, B, C^{\prime}\right)$ with $C^{\prime} \neq C$. Moreover, $C$ and $C^{\prime}$ are identical up to one element.

(iii) Or an element of $\{0,1\} \times \Delta(G)$.

Proof. See Appendix.

This theorem implies that, for almost every $W$ and $p$, for all pairs $(B, C) \in \mathcal{B} \times \mathcal{C}$, the set $\mathscr{L}(W, p, B, C)$ consists of a finite number of smooth arcs and loops, see Mas-Colell (1985). Theorem 3 does not claim that the set $\mathscr{L}(W, p, B, C)$ is non-empty. Since by definition the empty set qualifies as a $k$-dimensional manifold for any $k$, a 1-dimensional manifold with boundary may be empty.

Herings and Peeters (2001) develop a homotopy to compute the Nash equilibrium selected by the tracing procedure for finite normal-form games. The application of the tracing procedure to network formation problems involves two main differences. First, there is the nondifferentiability of $F_{i j}$, which we tackle by the introduction of the choice function $C$. Second, the perturbation argument that is needed to prove genericity is significantly more involved, caused by the fact that the utility of forming a link potentially depends on the payoffs of all $2^{|L|-1}$ networks where that link is present.

If $\mathscr{L}(W, p, B, C)$ has a boundary point in $(0,1) \times \Delta(G)$, then there is a unique pair $\left(B^{\prime}, C^{\prime}\right) \in \mathcal{B} \times \mathcal{C}$ such that $\mathscr{L}\left(W, p, B^{\prime}, C^{\prime}\right)$ and $\mathscr{L}(W, p, B, C)$ share this boundary point. The uniqueness of $\left(B^{\prime}, C^{\prime}\right)$ implies that the sets $\mathscr{L}(W, p, B, C)$ and $\mathscr{L}\left(W, p, B^{\prime}, C^{\prime}\right)$ are nicely 
linked to each other. Generically, it does not happen that three sets have a common boundary point, nor does it happen that a boundary point of one set intersects the interior of another set.

If $\mathscr{L}(W, p, B, C)$ has a boundary point in $\{0,1\} \times \Delta(G)$, then this point does not belong to any other set $\mathscr{L}\left(W, p, B^{\prime}, C^{\prime}\right)$. This implies that such a boundary point is also a boundary point of $\mathscr{L}(W, p)$.

Formally, the structure of $\mathscr{L}(W, p)$ is as follows.

Theorem 4. For an open set $(W, p) \in \mathcal{W} \times \Delta(G)$ with full Lebesgue measure, $\mathscr{L}(W, p)$ is a compact 1-dimensional piecewise $C^{\infty}$ manifold with boundary. The boundary of $\mathscr{L}(W, p)$ is given by the intersection of $\mathscr{L}(W, p)$ and $\{0,1\} \times \Delta(G)$. There is a unique boundary point in $\{0\} \times \Delta(G)$.

Proof. The first part of this theorem follows from Theorem 3 and the door-in door-out principle as described in Lemke and Howson (1964). For the second part, notice that $(0, x) \in \mathscr{L}(W, p)$ if and only if, for every $i j \in L, x_{i j} \in \arg \max _{s \in[0,1]} s F_{i j}\left(0, p_{-i j}\right)$. If there would be more than one optimal solution, then there is $i j \in L$ such that both $x_{i j}=0$ and $x_{i j}=1$ solves the problem $\max _{s \in[0,1]} s F_{i j}\left(0, p_{-i j}\right)$. This leads to a contraction with Theorem 3 .

Generically, the set $\mathscr{L}(W, p)$ consists of a finite number of arcs and loops. Although it is not necessarily the case that these arcs and loops are smooth, the number of non-differentiabilities is finite at most. Theorem 3 implies that all arcs in $\mathscr{L}(W, p)$ start and end in $\{0,1\} \times \Delta(G)$. Each such path consists of a finite sequence of smooth arcs of the sets $\mathscr{L}(W, p, B, C)$.

Generically, there is exactly one point of $\mathscr{L}(W, p)$ that belongs to $\{0\} \times \Delta(G)$. This point is a boundary point of $\mathscr{L}(W, p, B, C)$, where $B$ contains exactly one member for each link $i j \in L$. It holds that $s_{i j}^{0} \in B$ if given prior beliefs $p$ it is optimal not to form a link and $s_{i j}^{1} \in B$ if it is optimal to create a link. It holds that $C(i j)=i$ if $f_{i j}^{i}\left(p_{-i j}\right)<f_{i j}^{j}\left(p_{-i j}\right)$ and $C(i j)=j$ if $f_{i j}^{j}\left(p_{-i j}\right)<f_{i j}^{i}\left(p_{-i j}\right)$. By Theorem 4 this point is the starting point of a uniquely defined arc of $\mathscr{L}(W, p)$. This arc is the unique feasible path of $\mathscr{L}(W, p)$ that transforms prior beliefs into beliefs that correspond with a pairwise stable network.

Corollary 3. For an open set $(W, p) \in \mathcal{W} \times \Delta(G)$ with full Lebesgue measure, the linear tracing procedure is well-defined.

The set $\mathscr{L}(W, p) \cap(\{1\} \times \Delta(G))$ consists of all pairwise stable networks of $W$. Precisely one of these pairwise stable networks is an element of the unique feasible path of $\mathscr{L}(W, p)$. The remaining pairwise stable networks are pairwise connected by arcs from $\mathscr{L}(W, p)$, so the number of pairwise stable networks is odd.

Corollary 4. For an open set $(W, p) \in \mathcal{W} \times \Delta(G)$ with full Lebesgue measure, the number of pairwise stable networks is odd.

The generic oddness of the number of Nash equilibria in normal-form games is a wellknown result of Harsanyi (1973). The existence of a pairwise stable network is a recent result of Bich and Morhaim (2020). Corollary 4 shows that when agents have linear payoff functions, Bich and Morhaim's result can be sharpened to generic oddness. 


\section{A Differentiable Approach}

A potential drawback of the method of Section 4 is that one has to check whether a boundary point of $\mathscr{L}(W, p, B, C)$ is reached, and if yes, one has to switch to a different system of equalities defined by another pair $\left(B^{\prime}, C^{\prime}\right)$. This switching can be a serious problem in terms of computation time. There are $\prod_{i j \in L}\left(2^{2}-1\right)=3^{|L|}$ different sets $B$ and $2^{|L|}$ different functions $C$, whereas each one of them may be generated several times in the course of the algorithm. We show next that switching can be avoided by choosing an appropriate transformation of variables which results in an everywhere differentiable homotopy.

Let $i j \in L$. The constraints in (10)-(11) and (16)-(17) originate from the non-differentiability of $F_{i j}=\min \left\{F_{i j}^{i}, F_{i j}^{j}\right\}$ as defined in (9). We can avoid the min operator by observing that

$$
F_{i j}\left(t, x_{-i j}\right)=\min _{s \in[0,1]} s F_{i j}^{i}\left(t, x_{-i j}\right)+(1-s) F_{i j}^{j}\left(t, x_{-i j}\right), \quad\left(t, x_{-i j}\right) \in[0,1] \times[0,1]^{L \backslash\{i j\}} .
$$

The KKT conditions of this problem imply that $\sigma_{i j}^{i} \in[0,1]$ is a solution if and only if there exist real numbers $\sigma_{i j}^{j}, \mu_{i j}^{i}, \mu_{i j}^{j}$ such that

$$
\begin{aligned}
F_{i j}^{i}\left(t, x_{-i j}\right)-F_{i j}^{j}\left(t, x_{-i j}\right)+\mu_{i j}^{j}-\mu_{i j}^{i} & =0, \\
\mu_{i j}^{i} \sigma_{i j}^{i} & =0, \\
\mu_{i j}^{j} \sigma_{i j}^{j} & =0, \\
\sigma_{i j}^{i}+\sigma_{i j}^{i}-1 & =0, \\
\sigma_{i j}^{i}, \sigma_{i j}^{j}, \mu_{i j}^{i}, \mu_{i j}^{j} & \geq 0 .
\end{aligned}
$$

We can therefore replace the constraints in (10)-(11) and (16)-(17) by (24) and

$$
\sigma_{i j}^{i} F_{i j}^{i}\left(t, x_{-i j}\right)+\sigma_{i j}^{j} F_{i j}^{j}\left(t, x_{-i j}\right)+\alpha_{i j}-\beta_{i j}=0 .
$$

To obtain a differentiable homotopy, we introduce a transformation of variables, which is also used in Garcia and Zangwill (1981). For $\kappa=\left(\kappa_{i j}^{i}, \kappa_{i j}^{j}\right)_{i j \in L} \in \mathbb{R}^{2 L}$ and $\lambda=\left(\lambda_{i j}^{0}, \lambda_{i j}^{1}\right)_{i j \in L} \in$ $\mathbb{R}^{2 L}$, we define

$$
\begin{array}{ll}
\sigma_{i j}^{i}(\kappa)=\left(\max \left\{\kappa_{i j}^{i}, 0\right\}\right)^{2}, & \mu_{i j}^{i}(\kappa)=\left(\max \left\{-\kappa_{i j}^{i}, 0\right\}\right)^{2}, \\
\sigma_{i j}^{j}(\kappa)=\left(\max \left\{\kappa_{i j}^{j}, 0\right\}\right)^{2}, & \mu_{i j}^{j}(\kappa)=\left(\max \left\{-\kappa_{i j}^{j}, 0\right\}\right)^{2}, \\
x_{i j}(\lambda)=\left(\max \left\{\lambda_{i j}^{1}, 0\right\}\right)^{2}, & \alpha_{i j}(\lambda)=\left(\max \left\{-\lambda_{i j}^{1}, 0\right\}\right)^{2}, \\
y_{i j}(\lambda)=\left(\max \left\{\lambda_{i j}^{0}, 0\right\}\right)^{2}, & \beta_{i j}(\lambda)=\left(\max \left\{-\lambda_{i j}^{0}, 0\right\}\right)^{2} .
\end{array}
$$

After making these transformations of variables, we can use Theorem 2 to conclude that $(t, x) \in \mathscr{L}(W, p)$ if and only if $t \in[0,1]$ and, for every $i j \in L$, there are $\kappa_{i j}^{i}, \kappa_{i j}^{j}, \lambda_{i j}^{0}$, and $\lambda_{i j}^{1} \in \mathbb{R}$ such that

$$
\begin{aligned}
\sigma_{i j}^{i}(\kappa) F_{i j}^{i}\left(t, x_{-i j}(\lambda)\right)+\sigma_{i j}^{j}(\kappa) F_{i j}^{j}\left(t, x_{-i j}(\lambda)\right)+\alpha_{i j}(\lambda)-\beta_{i j}(\lambda) & =0, \\
F_{i j}^{i}\left(t, x_{-i j}(\lambda)\right)-F_{i j}^{j}\left(t, x_{-i j}(\lambda)\right)+\mu_{i j}^{j}(\kappa)-\mu_{i j}^{i}(\kappa) & =0, \\
\sigma_{i j}^{i}(\kappa)+\sigma_{i j}^{j}(\kappa)-1 & =0, \\
x_{i j}(\lambda)+y_{i j}(\lambda)-1 & =0 .
\end{aligned}
$$

Notice that all inequalities have disappeared and that the same system of equalities can be used, irrespective of the pair $(B, C) \in \mathcal{B} \times \mathcal{C}$. This is achieved by the specific construction of $\sigma_{i j}^{i}, \sigma_{i j}^{j}, \mu_{i j}^{i}, \mu_{i j}^{j}, x_{i j}, y_{i j}, \alpha_{i j}$, and $\beta_{i j}$ as functions of $\kappa$ and $\lambda$. 
Let $\mathscr{H}:[0,1] \times \mathbb{R}^{2 L} \times \mathbb{R}^{2 L} \rightarrow \mathbb{R}^{4 L}$ be the homotopy defined by the left-hand side of equalities (25). The homotopy $\mathscr{H}$ is continuously differentiable. It has the salient feature that its zeros describe the linear tracing procedure. Indeed, it holds that $(t, \kappa, \lambda) \in \mathscr{H}^{-1}(\{0\})$ if and only if $(t, x(\lambda)) \in \mathscr{L}(W, p)$.

The structure of $\mathscr{H}^{-1}(\{0\})$ is even simpler than the one of $\mathscr{L}(W, p)$ as the arcs and loops in $\mathscr{H}^{-1}(\{0\})$ are differentiable everywhere. The transformation of variables has smoothed out the kinks. As a direct consequence, it is possible to calculate the derivative at each point of the feasible path, which makes it possible to follow the path by means of easily implementable numerical methods.

\section{An Example for the Connections Model}

We apply our method to the connections model of Jackson and Wolinsky (1996) and illustrate how the linear tracing procedure works in selecting a pairwise stable network.

The connections model addresses social communication among individuals. Individuals communicate with those to whom they are linked and receive benefits from both direct and indirect links. The value from connections to other individuals depends on the shortest distance to these individuals. Consider a network $g \in G$. If $r_{i j}$ is the number of links in the shortest path between $i$ and $j$, then the benefits individual $i$ obtains from individual $j$ are given by $d^{r_{i j}}$, where $d \in(0,1)$ captures the idea that the value from indirect communication is discounted. The cost of maintaining a link are equal to $c$ for both involved individuals. The payoff of agent $i \in N$ in network $g \in G$ is therefore given by

$$
v^{i}(g)=\sum_{j \in N \backslash\{i\}} d^{r_{i j}}-\sum_{\{j \in N: i j \in g\}} c .
$$

As shown in Jackson and Wolinsky (1996), when $d-d^{2}<c<d$, a star encompassing all agents is pairwise stable. Since any agent can be the center of the star, there is multiplicity of pairwise stable networks. Moreover, other pairwise stable network architectures may coexist. It is then of interest to select one particular pairwise stable network via the linear tracing procedure with a specific prior $p$.

To illustrate, consider the case $n=3$. Figure 1 depicts the eight possible unweighted networks and Table 1 displays the corresponding payoffs.

Assume $d=0.8$ and $c=0.48$. Since the condition $d-d^{2}<c<d$ holds, the stars $g^{4}$, $g^{5}$, and $g^{6}$ are pairwise stable. There are also pairwise stable weighted networks, which can be discovered through the linear tracing procedure.

Let the prior be equal to $p=\left(p_{12}, p_{13}, p_{23}\right)=(0.7,0.8,0.9)$ and consider link 12 at $t=0$. If this link is not formed, then network $g^{0}$ results with probability $0.02, g^{2}$ with probability $0.08, g^{3}$ with probability 0.18 , and $g^{6}$ with probability 0.72 . The expected payoff for agent 1 is 0.7168 and it is 0.7488 for agent 2 . If link 12 is created, then network $g^{1}$ results with probability $0.02, g^{4}$ with probability $0.08, g^{5}$ with probability 0.18 , and $g^{7}$ with probability 

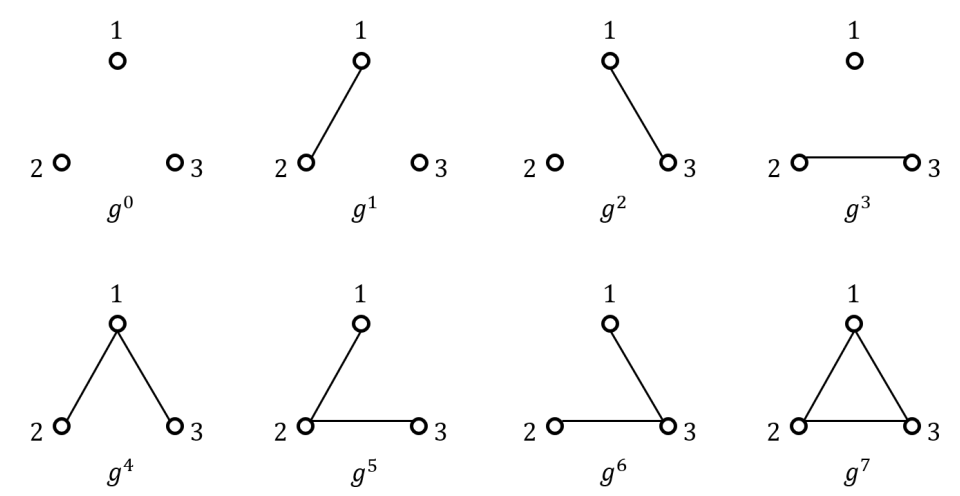

Figure 1: All unweighted networks with three agents

Table 1: Payoffs $v^{i}(g)$ in the connections model.

\begin{tabular}{c|ccc}
\hline$g$ & Agent 1 & Agent 2 & Agent 3 \\
\hline$g^{0}$ & 0 & 0 & 0 \\
$g^{1}$ & $d-c$ & $d-c$ & 0 \\
$g^{2}$ & $d-c$ & 0 & $d-c$ \\
$g^{3}$ & 0 & $d-c$ & $d-c$ \\
$g^{4}$ & $2(d-c)$ & $d+d^{2}-c$ & $d+d^{2}-c$ \\
$g^{5}$ & $d+d^{2}-c$ & $2(d-c)$ & $d+d^{2}-c$ \\
$g^{6}$ & $d+d^{2}-c$ & $d+d^{2}-c$ & $2(d-c)$ \\
$g^{7}$ & $2(d-c)$ & $2(d-c)$ & $2(d-c)$ \\
\hline
\end{tabular}

0.72. The expected payoff for agent 1 is 0.6912 and it is 0.6592 for agent 2 . Given these beliefs, agents 1 and 2 form link 12 with probability 0 . It holds that

$$
\begin{aligned}
& F_{12}^{1}\left(0, x_{-12}\right)=f_{12}^{1}\left(p_{-12}\right)=0.6912-0.7168=-0.0256 \\
& F_{12}^{2}\left(0, x_{-12}\right)=f_{12}^{2}\left(p_{-12}\right)=0.6592-0.7488=-0.0896 \\
& F_{12}\left(0, x_{-12}\right)=\min \left\{F_{12}^{1}\left(0, x_{-12}\right), F_{12}^{2}\left(0, x_{-12}\right)\right\}=-0.0896
\end{aligned}
$$

Using analogous calculations, it follows that $\min \left\{F_{13}^{1}\left(0, x_{-13}\right), F_{13}^{3}\left(0, x_{-13}\right)\right\}=F_{13}^{3}\left(0, x_{-13}\right)<$ 0 and $\min \left\{F_{23}^{2}\left(0, x_{-23}\right), F_{23}^{3}\left(0, x_{-23}\right)\right\}=F_{23}^{3}\left(0, x_{-23}\right)>0$. At $t=0$ there is a unique pairwise stable network $x \in \operatorname{PS}\left(W^{0}\right)$ given by $x=\left(x_{12}, x_{13}, x_{23}\right)=(0,0,1)$. The only $\left(B^{1}, C^{1}\right) \in$ $\mathcal{B} \times \mathcal{C}$ for which $\mathscr{L}\left(W, p, B^{1}, C^{1}\right)$ contains points with $t=0$ is given by $B^{1}=\left\{s_{12}^{0}, s_{13}^{0}, s_{23}^{1}\right\}$ and $C^{1}(12)=2, C^{1}(13)=3$, and $C^{1}(23)=3$, abbreviated as $C^{1}=(2,3,3)$.

We have that

$$
\begin{aligned}
F_{13}^{3}\left(t, x_{12}, x_{23}\right) & =t f_{13}^{3}\left(x_{12}, x_{23}\right)+(1-t) f_{13}^{3}\left(p_{12}, p_{23}\right) \\
& =t\left[d-c+d^{2} x_{12}\left(1-2 x_{23}\right)\right]+(1-t)\left[d-c+d^{2} p_{12}\left(1-2 p_{23}\right)\right] \\
& =d-c+d^{2}\left[t x_{12}\left(1-2 x_{23}\right)+(1-t) p_{12}\left(1-2 p_{23}\right)\right] .
\end{aligned}
$$

Since $d=0.8$ and $c=0.48$, at $x_{12}=0$ and $x_{23}=1$ the expression above reduces to

$$
\begin{aligned}
F_{13}^{3}(t, 0,1) & =0.32+0.64[(1-t) 0.7(-0.8)] \\
& =0.32(1.12 t-0.12) .
\end{aligned}
$$

Thus, when $t=3 / 28, F_{13}^{1}>F_{13}^{3}=0$, and the inequality $\alpha_{13} \geq 0$ in (20) becomes binding. A further continuation along the path without changing the set $B^{1}$ makes the value of $\alpha_{13}$ 
negative and thereby violates (20). None of the other inequality constraints becomes binding before $t=3 / 28$. As long as $t \leq \frac{3}{28}, \mathscr{L}\left(W, p, B^{1}, C^{1}\right)$ determines the feasible path.

To continue the linear tracing procedure, we expand $B^{1}$ by adding $s_{13}^{1}$ and define $B^{2}=$ $B^{1} \cup\left\{s_{13}^{1}\right\}$. Then, we can trace the arc in $\Gamma\left(W, p, B^{2}, C^{1}\right)$ from $(3 / 28,(0,0,1))$ to $(3 / 28,(0,1,1))$. Further continuation along the path would violate the upper bound of 1 on $x_{13}$ in inequality (18). We delete $s_{13}^{0}$ from $B^{2}$ by defining $B^{3}=B^{2} \backslash\left\{s_{13}^{0}\right\}$ and follow $\mathscr{L}\left(W, p, B^{3}, C^{1}\right)$ until $t=1$, where we obtain the pairwise stable network $x=(0,1,1)$ for the original network formation problem $W$. The path generated by the linear tracing procedure is summarized in Table 2.

Table 2: Tracing procedure via $\mathscr{L}(W, p, B, C)$

\begin{tabular}{llll}
\hline$t$ & $\mathscr{L}(W, p, B, C)$ & $B$ & $C$ \\
\hline$\left[0, \frac{3}{28}\right]\left\{(t,(0,0,1)) \mid t \in\left[0, \frac{3}{28}\right]\right\}$ & $\left\{s_{12}^{0}, s_{13}^{0}, s_{23}^{1}\right\}$ & $(2,3,3)$ \\
$\left\{\frac{3}{28}\right\}$ & $\left\{\left(\frac{3}{28},\left(0, x_{13}, 1\right)\right) \mid x_{13} \in[0,1]\right\}$ & $\left\{s_{12}^{0}, s_{13}^{0}, s_{13}^{1}, s_{23}^{1}\right\}$ & $(2,3,3)$ \\
{$\left[\frac{3}{28}, 1\right]$} & $\left\{(t,(0,1,1)) \mid t \in\left[\frac{3}{28}, 1\right]\right\}$ & $\left\{s_{12}^{0}, s_{13}^{1}, s_{23}^{1}\right\}$ & $(2,3,3)$ \\
\hline
\end{tabular}

The linear tracing procedure connects the pairwise stable network $(0,0,1)$ at $t=0$ to the pairwise stable network $(0,1,1)$ at $t=1$. A natural question is to which networks are the other pairwise stable stars at $t=1,(1,0,1)$ and $(0,1,1)$, connected? It turns out that they are connected to the pairwise stable networks $(0.5,0.5,1)$ and $(0.5,1,0.5)$ at $t=1$, respectively. One can easily verify that $x=(1,0.5,0.5)$ is also pairwise stable. Through the arcs in $\mathscr{L}(W, p)$, we find that $(1,(1,0.5,0.5))$ is connected to the symmetric pairwise stable network given by $x_{12}=x_{13}=x_{23}=\frac{1}{4}+\frac{1}{4} \sqrt{1+8 \frac{d-c}{d^{2}}}=0.809$ at $t=1$. These observations are summarized in Table 3.

\begin{tabular}{|c|c|c|c|c|}
\hline$t$ & $x$ & & $t$ & $x$ \\
\hline 0 & $(0,0,1)$ & $\longleftrightarrow$ & 1 & $(0,1,1)$ \\
\hline 1 & $(0.5,0.5,1)$ & $\longleftrightarrow$ & 1 & $(1,0,1)$ \\
\hline 1 & $(0.5,1,0.5)$ & $\longleftrightarrow$ & 1 & $(1,1,0)$ \\
\hline 1 & $(1,0.5,0.5)$ & $\longleftrightarrow$ & 1 & $(0.809,0.809,0.809)$ \\
\hline
\end{tabular}

In this example, there are seven pairwise stable networks. With prior $p=(0.7,0.8,0.9)$, the linear tracing procedure selects $(0,1,1)$, the star centered at agent 3 . The other six pairwise stable networks are pairwise connected.

\section{Numerical Implementation}

In this section we use the predictor-corrector method to numerically follow the solution path of the differentiable homotopy of Section 5. We study the connections model with four agents, 
with payoff functions given by

$$
v^{i}(g)=\sum_{j \in N \backslash\{i\}} d^{r_{i j}} w_{j}-\sum_{\{j \in N: i j \in g\}} c, \quad i \in N,
$$

where $w_{j} \in \mathbb{R}$ is the social value of agent $j$.

We assume that the agents have heterogeneous values given by $(0.8,0.9,1.1,1.2)$. As in Section 6, we take $d=0.8$ and $c=0.48$. For each $i j \in L$, the distance $r_{i j}$ can be computed by the well-known Dijkstra's algorithm. We then use the linear tracing procedure with several different priors. In particular, we consider $p^{1}, p^{2}, p^{3}$, and $p^{4}$ given by $p_{i j}^{1}=1$ for every $i j \in L$, $p^{2}=0.7 p^{1}, p^{3}=0.5 p^{1}$, and $p^{4}=0.3 p^{1}$.

We represent a network by $x=\left(x_{12}, x_{13}, x_{14}, x_{23}, x_{24}, x_{34}\right)$. The best response against prior $p^{1}$ is the empty network, $(0,0,0,0,0,0)$. The homotopy path goes from the empty network at $t=0$ to the network $(1,0,0,0.7630,0.7630,1)$ at $t=1$. With prior $p^{2}$, the path also starts from the empty network, but leads to the network $(1,0,0,1,0,1)$, a chain connecting all the agents. With prior $p^{3}$, the path starts from $(1,1,1,1,1,1)$, the complete network, and leads to $(1,0,0,1,0,1)$ as well. With prior $p^{4}$, the path also starts from the complete network, but leads to $(1,0,0,1,1,0)$, which is a star centered at agent 2 .

The paths resulting from priors $p^{1}$ and $p^{3}$ are plotted in Figure 2 and Figure 3, respectively, where the dashed lines indicate the variable $t$.

Table 4: Paths in $\mathscr{H}^{-1}(\{0\})$ with different priors

\begin{tabular}{llll}
\hline Prior & $\operatorname{PS}\left(W^{0}\right)$ & $\left(\lambda_{i j}^{1}\right)_{i j \in L}$ & Links formed \\
\hline $\mathbb{1}$ & 0 & $(1,-0.5219,-0.5219,0.8735,0.8735,1)$ & $\left\{x_{12}, x_{23}, x_{24}, x_{34}\right\}$ \\
$0.7 \cdot \mathbb{1}$ & 0 & $(1,-0.5933,-0.4996,1,-0.5367,1)$ & $\left\{x_{12}, x_{23}, x_{34}\right\}$ \\
$0.5 \cdot \mathbb{1}$ & $\mathbb{1}$ & $(1,-0.5933,-0.4996,1,-0.5367,1)$ & $\left\{x_{12}, x_{23}, x_{34}\right\}$ \\
$0.3 \cdot \mathbb{1}$ & $\mathbb{1}$ & $(1,-0.5933,-0.5933,1,1,-0.5514)$ & $\left\{x_{12}, x_{23}, x_{24}\right\}$ \\
\hline
\end{tabular}

We summarize the results obtained by tracing the paths with these priors in Table 4 . We use 11 to denote the vector of ones. The outcome selected by the linear tracing procedure depends on the prior and might even be different if the starting network is the same.

Next, we randomly generate 10 priors and find 8 different pairwise stable networks. Two of them have non-integer components and all of them are different from the solutions obtained above. This confirms that as in non-cooperative game theory, the multiplicity of pairwise stable networks is a serious problem, and that the specific computational method used is of crucial importance.

\section{Conclusion}

This paper studies the problem of computing a pairwise stable network as introduced in Bich and Morhaim (2020), extending work by Jackson and Wolinsky (1996). To do so, we first establish that finding a pairwise stable network is equivalent to finding a Nash equilibrium of a 


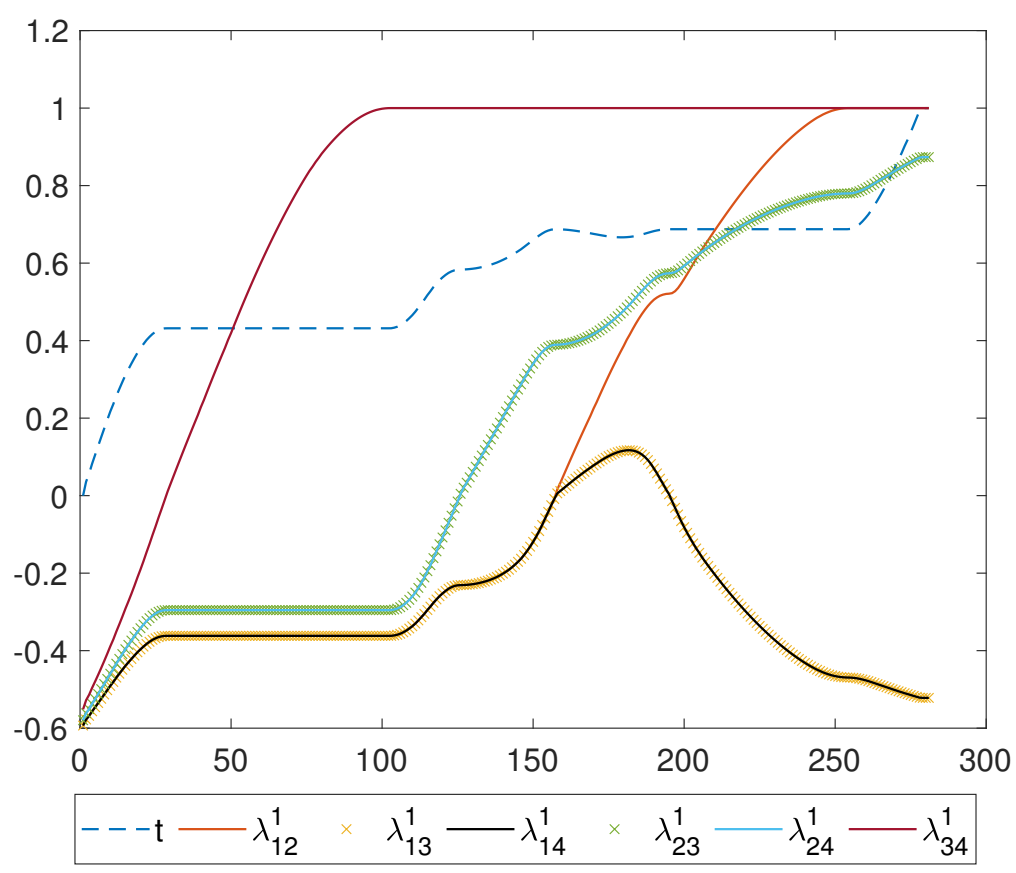

Figure 2: Tracing procedure with $p^{1}:\left(\lambda_{i j}^{1}\right)_{i j \in L}$ and $t$ versus iterations.

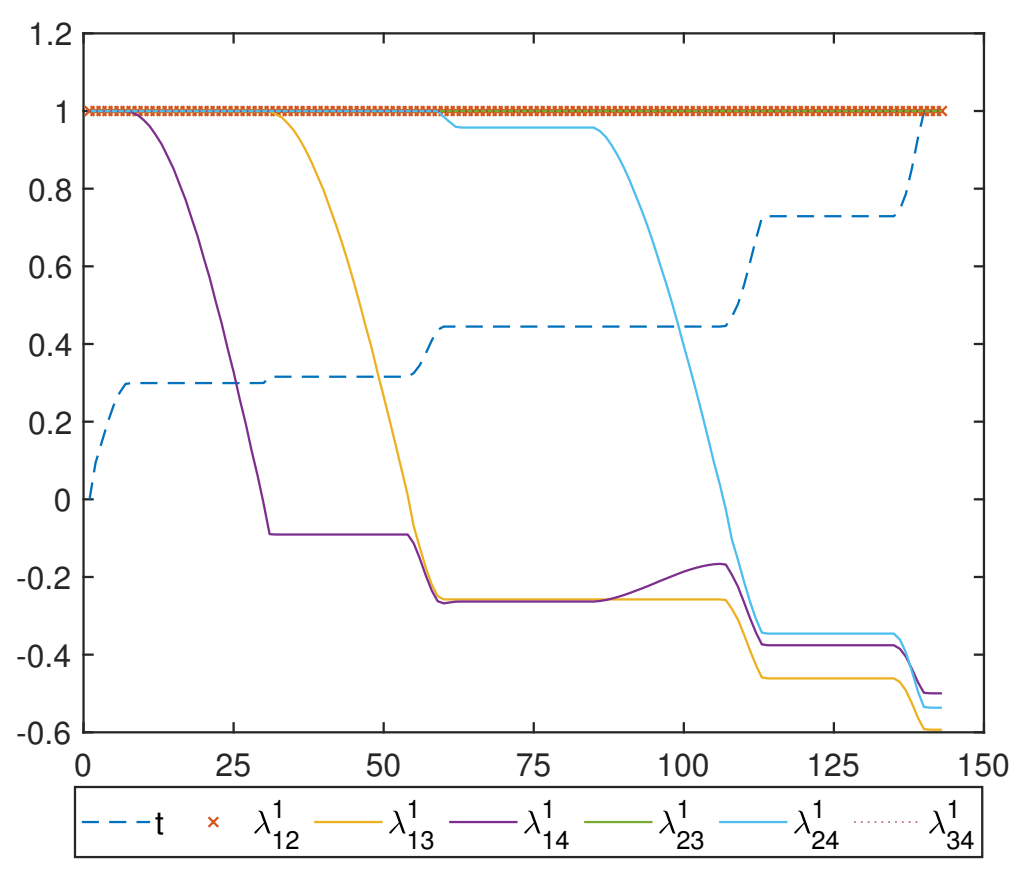

Figure 3: Tracing procedure with $p^{3}:\left(\lambda_{i j}^{1}\right)_{i j \in L}$ and $t$ versus iterations. 
game played by the links between every two agents. This allows us to extend the linear tracing procedure for normal-form games to network formation problems and select one particular pairwise stable network for any given prior belief. We show that generically the linear tracing procedure consists of a finite number of piecewise smooth arcs and loops. From this structure, we can conclude that the linear tracing procedure is well-defined for almost all network formation problems. As a by-product, it follows that for generic network formation problems the number of pairwise stable networks is odd, which sharpens the existence result of Bich and Morhaim (2020). An application of our method to the connections model of Jackson and Wolinsky (1996) reveals that there are many pairwise stable networks and that the selection of one particular pairwise stable network depends heavily on the initial beliefs of the agents.

\section{Appendix}

Before proving Theorem 3, we need some notations and definitions from the theory of regular constraint sets as presented in Jongen, Jonker, and Twilt (1983). For some $r \geq 1$ a subset $Y$ of $\mathbb{R}^{m}$ is called a $k$-dimensional $C^{r}$ manifold with generalized boundary (MGB), if for every $y \in Y$ there exists a $C^{r}$ diffeomorphism $\phi: U \rightarrow V$, where $U$ is an open subset of $\mathbb{R}^{m}$ containing $y$ and $V$ is open in $\mathbb{R}^{m}$, and some integer $\ell(y) \geq 0$, such that $\phi(y)=0$ and $\phi(U \cap Y)$ equals $\left\{z \in V \mid z_{i}=0, i=1, \ldots, m-k\right.$, and $\left.z_{i} \geq 0, i=m-k+1, \ldots, m-k+\ell(y)\right\}$. If for every element $y$ of an MGB $Y$ it holds that $\ell(y) \leq 1$, then $Y$ is called a manifold with boundary and the set of elements $y$ for which $\ell(y)=1$ is a $(k-1)$-dimensional manifold, called the boundary of $Y$.

Let $H^{1}$ and $H^{2}$ be two finite index sets and, for all $h \in H^{1}$, let $\gamma_{h}$ and, for all $h \in H^{2}, \eta_{h}$ be $C^{r}$ functions defined on some open subset $X$ of $\mathbb{R}^{m}$. We define

$$
Y[\gamma, \eta]=\left\{y \in X \mid \forall h \in H^{1}, \gamma_{h}(y)=0, \text { and }, \forall h \in H^{2}, \eta_{h}(y) \geq 0\right\}
$$

For every $y \in X$, we define the set $H^{0}(y)=\left\{h \in H^{2} \mid \eta_{h}(y)=0\right\}$. If for every $y \in Y[\gamma, \eta]$ it holds that

$$
\left\{\partial_{y} \gamma_{h}(y) \mid h \in H^{1}\right\} \cup\left\{\partial_{y} \eta_{h}(y) \mid h \in H^{0}(y)\right\}
$$

is a set of independent vectors, then $Y[\gamma, \eta]$ is called a $C^{r}$ regular constraint set (RCS). In Jongen, Jonker, and Twilt (1983) it is shown that every $C^{r} \mathrm{RCS}$ is an $\left(m-\left|H^{1}\right|\right)$-dimensional $C^{r}$ MGB such that, for every $y \in Y[\gamma, \eta], \ell(y)=\left|H^{0}(y)\right|$.

Fix a prior $p \in \Delta(G)$. For every tuple of utility functions $v$, for every $(B, C) \in \mathcal{B} \times \mathcal{C}$, we define the functions $\gamma^{B, C, v}: \mathbb{R}^{3|L|+1} \rightarrow \mathbb{R}^{3|L|}$ and $\eta^{B, C, v}: \mathbb{R}^{3|L|+1} \rightarrow \mathbb{R}^{3|L|+2}$ in such a way that $\gamma^{B, C, v}$ equals the left-hand side of the equalities (10)-(14) and $\eta^{B, C, v}$ the left-hand side of 
the inequalities (16)-(23),

$$
\gamma^{B, C, v}(t, x, \alpha, \beta)= \begin{cases}F_{i j}^{i}\left(t, x_{-i j}\right)+\alpha_{i j}-\beta_{i j}, & i j \in L, i \in C(i j), \\ F_{i j}^{J}\left(t, x_{-i j}\right)+\alpha_{i j}-\beta_{i j}, & i j \in L, j \in C(i j), \\ x_{i j}, & s_{i j}^{1} \notin B, \\ x_{i j}-1, & s_{i j}^{0} \notin B, \\ \beta_{i j}, & s_{i j}^{0} \in B, \\ \alpha_{i j}, & s_{i j}^{1} \in B,\end{cases}
$$

and

$$
\eta^{B, C, v}(t, x, \alpha, \beta)= \begin{cases}F_{i j}^{j}\left(t, x_{-i j}\right)-F_{i j}^{i}\left(t, x_{-i j}\right), & i j \in L, i \in C(i j), \\ F_{i j}^{i}\left(t, x_{-i j}\right)-F_{i j}^{j}\left(t, x_{-i j}\right), & i j \in L, j \in C(i j), \\ 1-x_{i j}, & s_{i j}^{0} \in B, \\ x_{i j}, & s_{i j}^{1} \in B, \\ \alpha_{i j}, & s_{i j}^{1} \notin B, \\ \beta_{i j}, & s_{i j}^{0} \notin B, \\ t, & \\ -t+1 . & \end{cases}
$$

A point $(t, x, \alpha, \beta) \in \mathbb{R}^{3|L|+1}$ is a solution of (10)-(23) if and only if $\gamma^{B, C, v}(t, x, \alpha, \beta)=0$ and $\eta^{B, C, v}(t, x, \alpha, \beta) \geq 0$.

For every $(B, C) \in \mathcal{B} \times \mathcal{C}$, we define the functions $\gamma^{B, C}: \mathbb{R}^{3|L|+1} \times \mathbb{R}^{n 2^{|L|}} \rightarrow \mathbb{R}^{3|L|}$ and $\eta^{B, C}: \mathbb{R}^{3|L|+1} \times \mathbb{R}^{n 2^{|L|}} \rightarrow \mathbb{R}^{3|L|+2}$ by specifying, for every $(t, x, \alpha, \beta, v) \in \mathbb{R}^{3|L|+1} \times \mathbb{R}^{n 2^{|L|}}$, $\gamma^{B, C}(t, x, \alpha, \beta, v)=\gamma^{B, C, v}(t, x, \alpha, \beta)$ and $\eta^{B, C}(t, x, \alpha, \beta, v)=\eta^{B, C, v}(t, x, \alpha, \beta)$. For convenience, we write $\gamma^{B, C}=\left(\gamma_{1}^{B, C}, \ldots, \gamma_{6}^{B, C}\right)$, where $\gamma_{1}^{B, C}=\left(\gamma_{1, i j}^{B, C}\right)_{i j \in L, i \in C(i j)}, \gamma_{2}^{B, C}=$ $\left(\gamma_{2, i j}^{B, C}\right)_{i j \in L, j \in C(i j)}, \gamma_{3}^{B, C}=\left(\gamma_{3, i j}^{B, C}\right)_{s_{i j}^{1} \notin B}, \gamma_{4}^{B, C}=\left(\gamma_{4, i j}^{B, C}\right)_{s_{i j}^{0} \notin B}, \gamma_{5}^{B, C}=\left(\gamma_{5, i j}^{B, C}\right)_{s_{i j}^{0} \in B}$, and $\gamma_{6}^{B, C}=$ $\left(\gamma_{6, i j}^{B, C}\right)_{s_{i j}^{1} \in B}$. We use analogous notational conventions for the functions $\gamma^{B, C, v}, \eta^{B, C}$, and $\eta^{B, C, v}$. The set of all indices related to $\gamma^{B, C}$ as well as $\gamma^{B, C, v}$ is denoted by $H^{1, B, C}$. The set of all indices related to $\eta^{B, C}$ as well as $\eta^{B, C, v}$ is denoted by $H^{2, B, C}$.

The structure of the proof of Theorem 3 is as follows. First, it is shown that for almost every $v \in \mathbb{R}^{n 2^{|L|}}$, for every $(B, C) \in \mathcal{B} \times \mathcal{C}, Y\left[\gamma^{B, C, v}, \eta^{B, c, v}\right]$ is a regular constraint set by showing that for every $y \in Y\left[\gamma^{B, C, v}, \eta^{B, C, v}\right]$,

$$
\left\{\partial_{y} \gamma_{h}^{B, C, v}(y) \mid h \in H^{1, B, C}\right\} \cup\left\{\partial_{y} \eta_{h}^{B, C, v}(y) \mid h \in H^{0, B, C}(y)\right\}
$$

is a set of independent vectors. Lemma 2 is used to handle points $y$ for which $H^{0, B, C}(y)=\emptyset$, Lemma 3 deals with points $y$ such that the cardinality of $H^{0, B, C}(y)$ is one, and Lemma 4 implies that generically $H^{0, B, C}(y)$ contains at most one element. Let the symbol $\pitchfork$ denote transversal intersection.

Lemma 2. Let $p \in \Delta(G)$ and $(B, C) \in \mathcal{B} \times \mathcal{C}$. Then, for almost every $v \in \mathbb{R}^{n 2^{|L|}}$, it holds that $\gamma^{B, C, v} \pitchfork\{0\}$.

Proof. Let $\bar{y}=(\bar{t}, \bar{x}, \bar{\alpha}, \bar{\beta}, \bar{v}) \in \mathbb{R}^{3|L|+1} \times \mathbb{R}^{n 2^{|L|}}$ be such that $\gamma^{B, C}(\bar{y})=0$.

We show first that the matrix of partial derivatives $P$ defined by

$$
P=\left[\begin{array}{l}
\partial_{v} \gamma_{1}^{B, C}(\bar{y}) \\
\partial_{v} \gamma_{2}^{B, C}(\bar{y})
\end{array}\right]
$$


has full row rank. Let $i j \in L$ be such that $i \in C(i j)$. We have that

$$
\begin{aligned}
\partial_{v} \gamma_{1, i j}^{B, C}(\bar{y})= & \partial_{v} F_{i j}^{i}\left(\bar{t}, \bar{x}_{-i j}\right) \\
= & \partial_{v}\left[\bar{t} f_{i j}^{i}\left(\bar{x}_{-i j}\right)+(1-\bar{t}) f_{i j}^{i}\left(p_{-i j}\right)\right] \\
= & \partial_{v} \bar{t} \sum_{g \in G} v^{i}(g)\left(2 g_{i j}-1\right) \pi_{g_{-i j}}\left(\bar{x}_{-i j}\right) \\
& +\partial_{v}(1-\bar{t}) \sum_{g \in G} v^{i}(g)\left(2 g_{i j}-1\right) \pi_{g_{-i j}}\left(p_{-i j}\right) \\
= & \partial_{v} \sum_{\left\{g \in G \mid g_{i j}=1\right\}}\left[\bar{t} \pi_{g_{-i j}}\left(\bar{x}_{-i j}\right)+(1-\bar{t}) \pi_{g_{-i j}}\left(p_{-i j}\right)\right] v^{i}(g) \\
& -\partial_{v} \sum_{\left\{g \in G \mid g_{i j}=0\right\}}\left[\bar{t} \pi_{g_{-i j}}\left(\bar{x}_{-i j}\right)+(1-\bar{t}) \pi_{g_{-i j}}\left(p_{-i j}\right)\right] v^{i}(g) .
\end{aligned}
$$

Let $g \in G$ be such that $g_{i j}=1$. It holds that

$$
\partial_{v^{i}(g)} \gamma_{1, i j}^{B, C}(\bar{y})=\bar{t} \pi_{g_{-i j}}\left(\bar{x}_{-i j}\right)+(1-\bar{t}) \pi_{g_{-i j}}\left(p_{-i j}\right) .
$$

Let $g \in G$ be such that $g_{i j}=0$. It holds that

$$
\partial_{v^{i}(g)} \gamma_{1, i j}^{B, C}(\bar{y})=-\bar{t} \pi_{g_{-i j}}\left(\bar{x}_{-i j}\right)-(1-\bar{t}) \pi_{g_{-i j}}\left(p_{-i j}\right) .
$$

Let $m^{i j} \in \mathbb{R}^{n 2^{|L|}}$ be the vector such that $m_{k, g}^{i j}=1$ if $k=i$ and $g_{i j}=1$, and $m_{k, g}^{i j}=0$ otherwise. It follows that

$$
\begin{aligned}
\partial_{v} \gamma_{1, i j}^{B, C}(\bar{y}) m^{i j} & =\sum_{\left\{g \in G \mid g_{i j}=1\right\}}\left[\bar{t} \pi_{g_{-i j}}\left(\bar{x}_{-i j}\right)+(1-\bar{t}) \pi_{g_{-i j}}\left(p_{-i j}\right)\right] \\
& =\bar{t} \sum_{\left\{g \in G \mid g_{i j}=1\right\}} \pi_{g_{-i j}}\left(\bar{x}_{-i j}\right)+(1-\bar{t}) \sum_{\left\{g \in G \mid g_{i j}=1\right\}} \pi_{g_{-i j}}\left(p_{-i j}\right) \\
& =\bar{t}+1-\bar{t}=1 .
\end{aligned}
$$

If $k \neq j$ is such that $i k \in L$ and $i \in C(i k)$, then

$$
\begin{aligned}
\partial_{v} \gamma_{1, i k}^{B, C}(\bar{y}) m^{i j}= & \left.\sum_{\left\{g \in G \mid g_{i j}=1\right.} \text { and } g_{i k}=1\right\} \\
& \left.-\sum_{\left\{g \in G \mid g_{i j}=1\right.} \text { and } g_{i k}=0\right\} \\
= & \left.\sum_{\left\{g \in G \mid g_{i j}=1\right\}}\left[\bar{t} \pi_{g_{-i k}}\left(\bar{x}_{-i k}\right)+(1-\bar{t}) \pi_{g_{-i k}}\left(\bar{x}_{-i k}\right)\right]+(1-\bar{t}) \pi_{g_{-i k}}\left(p_{-i k}\right)\right] \\
& \left.-\bar{t} \pi_{g_{-i k}}\left(\bar{x}_{-i k}\right)-(1-\bar{t}) \pi_{g_{-i k}}\left(p_{-i k}\right)\right] \\
= & 0 .
\end{aligned}
$$

Finally, for every $k \ell \in L$ such that $i \neq k \in C(k \ell)$, it holds that

$$
\partial_{v} \gamma_{1, k \ell}^{B, C}(\bar{y}) m^{i j}=\sum_{\left\{g \in G \mid g_{i j}=1\right\}} \partial_{v^{i}(g)} \gamma_{1, k \ell}^{B, C}(\bar{y})=0
$$

where the last equality follows from $k \neq i$. It follows by symmetry that, for every $k \ell \in L$ such that $\ell \in C(k \ell)$,

$$
\partial_{v} \gamma_{2, k \ell}^{B, C}(\bar{y}) m^{i j}=0 .
$$

Now let $i j \in L$ be such that $j \in C(i j)$. Let $m^{i j} \in \mathbb{R}^{n 2^{|L|}}$ be the vector such that $m_{k, g}^{i j}=1$ if $k=j$ and $g_{i j}=1$, and $m_{k, g}^{i j}=0$ otherwise. It follows by symmetry that

$$
\begin{aligned}
& \partial_{v} \gamma_{2, i j}^{B, C}(\bar{y}) m^{i j}=1, \\
& \partial_{v} \gamma_{2, k j}^{B, C}(\bar{y}) m^{i j}=0, \quad k j \in L, j \in C(k j), k \neq i, \\
& \partial_{v} \gamma_{2, k \ell}^{B, C}(\bar{y}) m^{i j}=0, \quad k \ell \in L, j \neq \ell \in C(k \ell), \\
& \partial_{v} \gamma_{1, k \ell}^{B, C}(\bar{y}) m^{i j}=0, \quad k \ell \in L, k \in C(k \ell) .
\end{aligned}
$$

Let $M$ be the $n 2^{|L|} \times|L|$-matrix with columns equal to $\left(m^{i j}\right)_{i j \in L, i \in C(i j)}$ and $\left(m^{i j}\right)_{i j \in L, j \in C(i j)}$. Our derivations show that

$$
P M=I,
$$


where $I$ is the $|L| \times|L|$ identity matrix. Since $M$ is a right-inverse of $P$, we have shown that $P$ has full row rank.

Let $z \in \mathbb{R}^{3|L|+1}$ be such that $z^{\top} \partial_{y} \gamma^{B, C}(\bar{y})=0$. Since $P$ has full row rank and $\partial_{v} \gamma_{3}^{B, C}(\bar{y})$, $\ldots, \partial_{v} \gamma_{6}^{B, C}(\bar{y})$ are matrices filled with zeros, it holds that $z_{1}=0$ and $z_{2}=0$.

Denote $\partial_{\left(x_{i j}\right)_{s_{i j}^{1} \notin B},\left(x_{i j}\right)_{s_{i j}^{0} \notin B}}$ by $\partial_{\widetilde{x}}$. We have that

$$
\begin{aligned}
z^{\top} \partial_{\widetilde{x}} \gamma^{B, C}(\bar{y}) & =z_{3}^{\top} \partial_{\widetilde{x}} \gamma_{3}^{B, C}(\bar{y})+z_{4}^{\top} \partial_{\widetilde{x}} \gamma_{4}^{B, C}(\bar{y})+z_{5}^{\top} \partial_{\widetilde{x}} \gamma_{5}^{B, C}(\bar{y})+z_{6}^{\top} \partial_{\widetilde{x}} \gamma_{6}^{B, C}(\bar{y}) \\
& =z_{3}^{\top} \partial_{\widetilde{x}} \gamma_{3}^{B, C}(\bar{y})+z_{4}^{\top} \partial_{\widetilde{x}} \gamma_{4}^{B, C}(\bar{y}) \\
& =\left(z_{3}^{\top}, z_{4}^{\top}\right) I=0,
\end{aligned}
$$

where the first equality follows from $z_{1}=0$ and $z_{2}=0$ and the second equality since $\gamma_{5}^{B, C}$ and $\gamma_{6}^{B, C}$ do not involve $x$. Since $I$ is the $(2|L|-|B|) \times(2|L|-|B|)$ identity matrix, we have that $z_{3}=0$ and $z_{4}=0$.

Denote $\partial_{\left(\beta_{i j}\right)_{s_{i j}^{0} \in B}}$ by $\partial_{\widetilde{\beta}}$. We have that

$$
\begin{aligned}
z^{\top} \partial_{\widetilde{\beta}} \gamma^{B, C}(\bar{y}) & =z_{5}^{\top} \partial_{\widetilde{\beta}} \gamma_{5}^{B, C}(\bar{y})+z_{6}^{\top} \partial_{\widetilde{\beta}} \gamma_{6}^{B, C}(\bar{y}) \\
& =z_{5}^{\top} \partial_{\widetilde{\beta}} \gamma_{5}^{B, C}(\bar{y}) \\
& =z_{5}^{\top} I=0,
\end{aligned}
$$

where $I$ is the $k \times k$ identity matrix with $k$ the cardinality of the set $\left\{i j \in L \mid s_{i j}^{0} \in B\right\}$. It follows that $z_{5}=0$.

Finally, denote $\partial_{\left(\alpha_{i j}\right)_{s_{i j}^{1} \in B}}$ by $\partial_{\widetilde{\alpha}}$. We have

$$
\begin{aligned}
z^{\top} \partial_{\widetilde{\alpha}} \gamma^{B, C}(\bar{y}) & =z_{6}^{\top} \partial_{\widetilde{\alpha}} \gamma_{6}^{B, C}(\bar{y}) \\
& =z_{6}^{\top} I=0,
\end{aligned}
$$

where $I$ is the $k \times k$ identity matrix with $k$ the cardinality of the set $\left\{i j \in L \mid s_{i j}^{1} \in B\right\}$. It follows that $z_{6}=0$.

We have shown that $z^{\top} \partial_{y} \gamma^{B, C}(\bar{y})=0$ implies $z=0$, so $\partial_{y} \gamma^{B, C}(\bar{y})$ has full row rank, and therefore $\gamma^{B, C}$ 历 $\{0\}$.

By the transversality theorem, see Mas-Colell (1985), Theorem I.2.2, and since $\gamma^{B, C}$ is a $C^{\infty}$ function, it follows that the complement of $\left\{v \in \mathbb{R}^{n 2^{|L|}} \mid \gamma^{B, C, v} \pitchfork\{0\}\right\}$ has Lebesgue measure zero.

Lemma 3. Let $p \in \Delta(G),(B, C) \in \mathcal{B} \times \mathcal{C}$, and $h \in H^{2, B, C}$. Then, for almost every $v \in \mathbb{R}^{n 2^{|L|}}$, it holds that $\left(\gamma^{B, C, v}, \eta_{h}^{B, C, v}\right) 历\{0\}$.

Proof. Let $\bar{y}=(\bar{t}, \bar{x}, \bar{\alpha}, \bar{\beta}, \bar{v}) \in \mathbb{R}^{3|L|+1} \times \mathbb{R}^{n 2^{|L|}}$ be such that $\gamma^{B, C}(\bar{y})=0$ and $\eta_{h}^{B, C}(\bar{y})=0$. We show that the matrix of partial derivatives of $\left(\gamma^{B, C}, \eta_{h}^{B, C}\right)$ at $\bar{y}$ has full row rank.

Consider the case where $h=(1, i j)$ for $i j \in L$ such that $i \in C(i j)$. Let $e^{i j} \in \mathbb{R}^{n 2^{|L|}}$ be the vector such that $e_{k, g}^{i j}=1$ if $k=j$ and $g_{i j}=1$, and $e_{k, g}^{i j}=0$ otherwise. Using the same arguments as in Lemma 2, it follows that $\partial_{v} \gamma_{1}^{B, C}(\bar{y}) e^{i j}=0$ and $\partial_{v} \gamma_{2}^{B, C}(\bar{y}) e^{i j}=0$. Also, repeating the arguments in Lemma 2, we have that

$$
\begin{aligned}
\partial_{v} \eta_{h}^{B, C}(\bar{y}) e^{i j} & =\partial_{v} F_{i j}^{j}\left(\bar{t}, \bar{x}_{-i j}\right) e^{i j}-\partial_{v} F_{i j}^{i}\left(\bar{t}, \bar{x}_{-i j}\right) e^{i j}=1, \\
\partial_{v} \eta_{h}^{B, C}(\bar{y}) m^{i j} & =\partial_{v} F_{i j}^{j}\left(\bar{t}, \bar{x}_{-i j}\right) m^{i j}-\partial_{v} F_{i j}^{i}\left(\bar{t}, \bar{x}_{-i j}\right) m^{i j}=-1 .
\end{aligned}
$$


For any other column $m$ of the matrix $M$, we have $\partial_{v} \eta_{h}^{B, C}(\bar{y}) m=0$. We conclude that

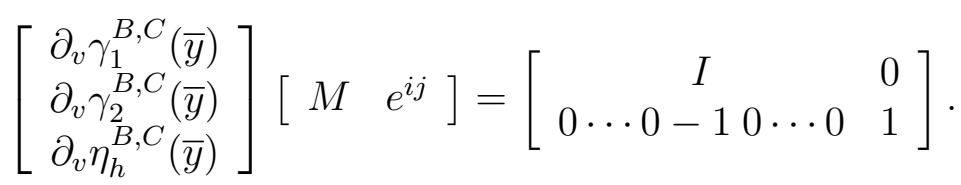

Since the matrix on the right-hand size has full rank, we can proceed as in Lemma 2 and conclude that the matrix of partial derivatives of $\left(\gamma^{B, C}, \eta_{h}^{B, C}\right)$ at $\bar{y}$ has full row rank.

The same argument applies if $h=(2, i j)$ for $i j \in L$ such that $j \in C(i j)$.

Consider $h=(3, i j)$, so $1-\bar{x}_{i j}=0$ and $s_{i j}^{0} \in B$. It follows that $s_{i j}^{1} \in B$, since otherwise (12) implies $\bar{x}_{i j}=0$, leading to a contradiction with $1-\bar{x}_{i j}=0$. Similarly, if $h=(4, i j)$, we have $\bar{x}_{i j}=0, s_{i j}^{1} \in B$, and $s_{i j}^{0} \in B$. Denote $\partial_{\left(x_{k \ell}\right)_{s_{k \ell}^{1} \notin B},\left(x_{k \ell}\right)_{s_{k \ell}^{0} \notin B}, x_{i j}}$ by $\partial_{\widetilde{x}}$. We have that

$$
\left[\begin{array}{l}
\partial_{\widetilde{x}} \gamma_{3}^{B, C}(\bar{y}) \\
\partial_{\widetilde{x}} \gamma_{4}^{B, C}(\bar{y}) \\
\partial_{\widetilde{x}} \eta_{h}^{B, C}(\bar{y})
\end{array}\right]=I
$$

The rest of the argument is identical to the one in the proof of Lemma 2.

Consider $h=(5, i j)$, so $s_{i j}^{1} \notin B$. Denote $\partial_{\left(\alpha_{k \ell}\right)_{s_{k \ell}^{1} \in B}, \alpha_{i j}}$ by $\partial_{\widetilde{\alpha}}$. We have that

$$
\left[\begin{array}{l}
\partial_{\widetilde{\alpha}} \gamma_{6}^{B, C}(\bar{y}) \\
\partial_{\widetilde{\alpha}} \eta_{h}^{B, C}(\bar{y})
\end{array}\right]=I
$$

The rest of the argument is identical to the one in the proof of Lemma 2.

Consider $h=(6, i j)$, so $s_{i j}^{0} \notin B$. Denote $\partial_{\left(\beta_{k \ell}\right)_{s_{k \ell}^{0} \in B}, \beta_{i j}}$ by $\partial_{\widetilde{\beta}}$. We have that

$$
\left[\begin{array}{l}
\partial_{\widetilde{\beta}} \gamma_{5}^{B, C}(\bar{y}) \\
\partial_{\widetilde{\beta}} \eta_{h}^{B, C}(\bar{y})
\end{array}\right]=I .
$$

The rest of the argument is identical to the one in the proof of Lemma 2.

Consider $h=7$. We start the argument as in the proof of Lemma 2 and complete it by the observation that $\partial_{t} \eta_{h}^{B, C}(\bar{y})=1$. The same argument works for $h=8$, where now $\partial_{t} \eta_{h}^{B, C}(\bar{y})=$ -1 .

We have shown that $\left(\gamma^{B, C}, \eta_{h}^{B, C}\right) 历\{0\}$. By the transversality theorem it follows that the complement of $\left\{v \in \mathbb{R}^{n 2^{|L|}} \mid\left(\gamma^{B, C, v}, \eta_{h}^{B, C, v}\right) 历\{0\}\right\}$ has Lebesgue measure zero.

Lemma 4. Let $p \in \Delta(G),(B, C) \in \mathcal{B} \times \mathcal{C}$, and $h, h^{\prime} \in H^{2, B, C}$ with $h \neq h^{\prime}$. Then, for almost every $v \in \mathbb{R}^{n 2^{|L|}}$, it holds that $\left(\gamma^{B, C, v}, \eta_{h}^{B, C, v}, \eta_{h^{\prime}}^{B, C, v}\right) 历\{0\}$.

Proof. Let $\bar{y}=(\bar{t}, \bar{x}, \bar{\alpha}, \bar{\beta}, \bar{v}) \in \mathbb{R}^{3|L|+1} \times \mathbb{R}^{n 2^{|L|}}$ be such that $\gamma^{B, C}(\bar{y})=0, \eta_{h}^{B, C}(\bar{y})=0$, and $\eta_{h^{\prime}}^{B, C}(\bar{y})=0$. We show that the matrix of partial derivatives of $\left(\gamma^{B, C}, \eta_{h}^{B, C}, \eta_{h^{\prime}}^{B, C}\right)$ at $\bar{y}$ has full row rank.

Consider the case where $h=(1, i j)$ for $i j \in L$ such that $i \in C(i j)$ and $h^{\prime}=\left(1, i^{\prime} j^{\prime}\right)$ for $i^{\prime} j^{\prime} \in L$ such that $i^{\prime} \in C\left(i^{\prime} j^{\prime}\right)$. Let $e^{i^{\prime} j^{\prime}} \in \mathbb{R}^{n 2^{|L|}}$ be the vector such that $e_{k, g}^{i^{\prime} j^{\prime}}=1$ if $k=i^{\prime}$ and 
$g_{i^{\prime} j^{\prime}}=1$, and $e_{k, g}^{i^{\prime} j^{\prime}}=0$ otherwise. Using the same arguments as in Lemma 3, we find that

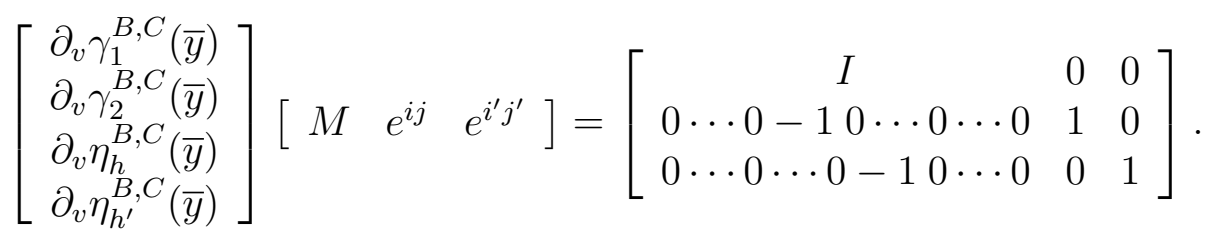

Since the matrix on the right-hand size has full rank, we can proceed as in Lemma 3 to show that the matrix of partial derivatives of $\left(\gamma^{B, C}, \eta_{h}^{B, C}, \eta_{h^{\prime}}^{B, C}\right)$ at $\bar{y}$ has full row rank.

The case where $h=(1, i j)$ for $i j \in L$ such that $i \in C(i j)$ and $h^{\prime}=\left(1, i^{\prime} j^{\prime}\right)$ for $i^{\prime} j^{\prime} \in L$ such that $j^{\prime} \in C\left(i^{\prime} j^{\prime}\right)$ as well as the case where $h=(1, i j)$ for $i j \in L$ such that $j \in C(i j)$ and $h^{\prime}=\left(1, i^{\prime} j^{\prime}\right)$ for $i^{\prime} j^{\prime} \in L$ such that $j^{\prime} \in C\left(i^{\prime} j^{\prime}\right)$ follow similarly. All other cases can be handled as in the proof of Lemma 3, except the case $h=7$ and $h^{\prime}=8$. However, that case implies $\bar{t}=0$ and $-\bar{t}+1=0$, so cannot occur.

We have shown that $\left(\gamma^{B, C}, \eta_{h}^{B, C}, \eta_{h^{\prime}}^{B, C}\right) 历\{0\}$. By the transversality theorem it follows that the complement of $\left\{v \in \mathbb{R}^{n 2^{|L|}} \mid\left(\gamma^{B, C, v}, \eta_{h}^{B, C, v}\right) \pitchfork\{0\}\right\}$ has Lebesgue measure zero.

Now we are ready to prove Theorem 3.

Proof. Fix a prior $p \in \Delta(G)$ and a pair $(B, C) \in \mathcal{B} \times \mathcal{C}$. The set $V$ consisting of $v \in \mathbb{R}^{n 2^{|L|}}$ such that $\gamma^{B, C, v} \pitchfork\{0\}$, for every $h \in H^{2, B, C},\left(\gamma^{B, C, v}, \eta_{h}^{B, C, v}\right) \pitchfork\{0\}$, and for every $h, h^{\prime} \in H^{2, B, C}$ with $h \neq h^{\prime},\left(\gamma^{B, C, v}, \eta_{h}^{B, C, v}, \eta_{h^{\prime}}^{B, C, v}\right) 历\{0\}$ has full Lebesgue measure by Lemmas 2, 3, and 4 .

Consider any $v \in V$. We show that $Y\left[\gamma^{B, C, v}, \eta^{B, C, v}\right]$ is a regular constraint set. Consider any $\bar{y} \in Y\left[\gamma^{B, C, v}, \eta^{B, C, v}\right]$. It has to be shown that

$$
\left\{\partial_{y} \gamma_{h}^{B, C, v}(\bar{y}) \mid h \in H^{1, B, C}\right\} \cup\left\{\partial_{y} \eta_{h}^{B, C, v}(\bar{y}) \mid h \in H^{0, B, C}(\bar{y})\right\}
$$

is a set of independent vectors. This is a consequence of Lemma 2 when $H^{0}(\bar{y})=\emptyset$ and a consequence of Lemma 3 when $\left|H^{0}(\bar{y})\right|=1$. Suppose $\left|H^{0}(\bar{y})\right| \geq 2$ and choose two elements, say $h$ and $h^{\prime}$, from $H^{0}(\bar{y})$. It follows from Lemma 4 that

$$
\left\{\partial_{y} \gamma_{h}^{B, C, v}(\bar{y}) \mid h \in H^{1, B, C}\right\} \cup\left\{\partial_{y} \eta_{h}^{B, C, v}(\bar{y}), \partial_{y} \eta_{h^{\prime}}^{B, C, v}(\bar{y})\right\}
$$

is a set of independent vectors, which leads to a contradiction as the set contains $3|L|+2$ vectors of dimension $3|L|+1$. Consequently, $\left|H^{0}(\bar{y})\right| \leq 1$, and $Y\left[\gamma^{B, C, v}, \eta^{B, C, v}\right]$ is a regular constraint set.

It follows that $\mathscr{O}(W, p, B, C)$ is a 1-dimensional manifold with boundary and that the boundary of $\mathscr{O}(W, p, B, C)$ is given by the elements $\bar{y} \in Y\left[\gamma^{B, C, v}, \eta^{B, C, v}\right]$ with $\left|H^{0, B, C}(\bar{y})\right|=$ 1. Therefore, a point in $\mathscr{O}(W, p, B, C)$ is a boundary point if and only if exactly one of the inequalities in (16)-(23) holds with equality. These properties carry over to $\mathscr{L}(W, p, B, C)$ since it is diffeomorphic to $\mathscr{O}(W, p, B, C)$ by Theorem 2 .

If the inequality $x_{i j} \geq 0$ (respectively, $x_{i j} \leq 1$ ) is binding, it holds that $x_{i j}=0$ (respectively, $\left.x_{i j}=1\right)$ and this boundary point is also a boundary point of $\mathscr{L}\left(W, p, B^{\prime}, C\right)$, where $B^{\prime}=B \backslash\left\{s_{i j}^{1}\right\}$ (respectively, $B^{\prime}=B \backslash\left\{s_{i j}^{0}\right\}$ ). If the inequality $\alpha_{i j} \geq 0$ (respectively, $\beta_{i j} \geq 0$ ) 
is binding, it holds that $\alpha_{i j}=0$ (respectively, $\beta_{i j}=0$ ) and this boundary point is also a boundary point of $\mathscr{L}\left(W, p, B^{\prime}, C\right)$, where $B^{\prime}=B \cup\left\{s_{i j}^{1}\right\}$ (respectively, $B^{\prime}=B \cup\left\{s_{i j}^{0}\right\}$ ). Alternative (i) holds.

If the inequality $F_{i j}^{j}\left(t, x_{-i j}\right)-F_{i j}^{i}\left(t, x_{-i j}\right) \geq 0$ is binding, this boundary point is also a boundary point of $\mathscr{L}\left(W, p, B, C^{\prime}\right)$, where $C^{\prime}(i j)=j$ and $C^{\prime}$ coincides with $C$ otherwise. If the inequality $F_{i j}^{j}\left(t, x_{-i j}\right)-F_{i j}^{i}\left(t, x_{-i j}\right) \geq 0$ is binding, this boundary point is also a boundary point of $\mathscr{L}\left(W, p, B, C^{\prime}\right)$, where $C^{\prime}(i j)=i$ and $C^{\prime}$ coincides with $C$ otherwise. Alternative (ii) holds.

If the binding inequality is $t \geq 0$ or $-t+1 \geq 0$, then alternative (iii) holds.

Compactness of $\mathscr{L}(W, p, B, C)$ is obvious.

It remains to be shown that there is an open set of network formation problems and priors with full Lebesgue measure for which the asserted properties hold. Let $Q$ be the set $\mathbb{R}^{n 2^{|L|}} \times$ $\Delta(G)$ of all network formation problems and priors and, for every $(B, C) \in \mathcal{B} \times \mathcal{C}$, let $Q^{B, C}$ be the subset of $Q$ consisting of all pairs $(v, p)$ for which the transversality statements in Lemma 2, Lemma 3, and Lemma 4 hold. It has already been shown that $Q \backslash Q^{B, C}$ has Lebesgue measure zero. If $(v, p) \in Q \backslash Q^{B, C}$, then there exists $y=(t, x, \alpha, \beta) \in \mathbb{R}^{3|L|+1}$ such that $(v, p, y)$ belongs to the set $R^{B, C}$ which is defined as the union of the sets

$$
\begin{aligned}
& \begin{cases}(v, p, y) \in Q \times \mathbb{R}^{3|L|+1} & \left.\mid \gamma^{B, C, v, p}(y)=0 \text { and } \operatorname{rank}\left(\partial_{y} \gamma^{B, C, v, p}(y)\right) \leq 3|L|-1\right\}, \\
(v, p, y) \in Q \times \mathbb{R}^{3|L|+1} & \mid \gamma^{B, C, v, p}(y)=0, \eta_{h}^{B, C, v, p}(y)=0,\end{cases} \\
& \left.\operatorname{rank}\left(\begin{array}{c}
\partial_{y} \gamma^{B, C, v, p}(y) \\
\partial_{y} \eta_{h}^{B, C, v, p}(y)
\end{array}\right) \leq 3|L|\right\}, \quad h \in H^{2, B, C} \\
& \left\{(v, p, y) \in Q \times \mathbb{R}^{3|L|+1} \mid \gamma^{B, C, v, p}(y)=0, \eta_{h}^{B, C, v, p}(y)=0, \eta_{h^{\prime}}^{B, C, v, p}(y)=0,\right. \\
& \left.\operatorname{rank}\left(\begin{array}{c}
\partial_{y} \gamma^{B, C, v, p}(y) \\
\partial_{y} \eta_{h}^{B, C, v, p}(y) \\
\partial_{y} \eta_{h^{\prime}}^{B, C, v, p}(y)
\end{array}\right) \leq 3|L|+1\right\}, \quad h, h^{\prime} \in H^{2, B, C}, h \neq h^{\prime} .
\end{aligned}
$$

The notation $\gamma^{B, C, v, p}$ and $\eta^{B, C, v, p}$ makes the dependence on the prior $p$ explicit. The set $R^{B, C}$ is closed since it is obtained by finite unions and intersections of closed sets. We define the projection $q: R^{B, C} \rightarrow Q$ by

$$
q(v, p, y)=(v, p), \quad(v, p, y) \in R^{B, C} .
$$

It holds that $q\left(R^{B, C}\right)=Q \backslash Q^{B, C}$. We show that $q$ is proper, i.e., that $q^{-1}(K)$ is compact if $K$ is a compact subset of $Q$. Let $K$ be a compact subset of $Q$. Clearly, $q^{-1}(K)$ is a closed subset of the set $R^{B, C}$, which is easily seen to be bounded, and therefore $q^{-1}(K)$ is compact. Since the image by a continuous proper mapping of a closed set is closed, it follows that $q\left(R^{B, C}\right)=Q \backslash Q^{B, C}$ is closed, so $Q^{B, C}$ is open. Since there is a finite number of pairs $(B, C) \in \mathcal{B} \times \mathcal{C}$, the intersection of the sets $Q^{B, C}$ over all $(B, C) \in \mathcal{B} \times \mathcal{C}$ is an open set of full Lebesgue measure of games and priors with the properties as stated in the theorem. 


\section{References}

Bich, P., AND L. Morhaim (2020), "On the Existence of Pairwise Stable Weighted Networks," forthcoming in Mathematics of Operations Research.

BlOCH, F., AND M.O. JACKSON (2006), "Definitions of Equilibrium in Network Formation Games," International Journal of Game Theory, 34, 305-318.

CAlvó-Armengol, A., AND R. İLKILIÇ (2009), "Pairwise-stability and Nash Equilibria in Network Formation," International Journal of Game Theory, 38, 51-79.

Chakrabarti, S., And R.P. Gilles (2007), "Network Potentials," Review of Economic Design, 11, 13-52.

EAves, B.C., And K. Schmedders (1999), "General Equilibrium Models and Homotopy Methods," Journal of Economic Dynamics and Control, 23, 1249-1279.

Garcia, C.B., And W.I. Zangwill (1981), Pathways to Solutions, Fixed Points, and Equilibria, Prentice-Hall Series in Computational Mathematics, Prentice-Hall, Englewood Cliffs.

Govindan, S., AND R. WiLson (2003), "A Global Newton Method to Compute Nash Equilibria," Journal of Economic Theory, 110, 65-86.

Govindan, S., AND R. Wilson (2009), “Global Newton Method for Stochastic Games," Journal of Economic Theory, 144, 414-421.

HARSANYI, J.C. (1973), “Oddness of the Number of Equilibrium Points," International Journal of Game Theory, 2, 235-250.

Harsanyi, J.C., And R. Selten (1988), A General Theory of Equilibrium Selection in Games, MIT Press, Cambridge.

Hellmann, T. (2013), "On the Existence and Uniqueness of Pairwise Stable Networks," International Journal of Game Theory, 42, 211-237.

Herings, P.J.J., AND R.J.A.P. PeEters (2001), "A Differentiable Homotopy to Compute Nash Equilibria of $n$-Person Games," Economic Theory, 18, 159-185.

HERINGS, P.J.J., AND R.J.A.P. PEETERs (2004), "Stationary Equilibria in Stochastic Games: Structure, Selection, and Computation," Journal of Economic Theory, 118, 32-60.

Herings, P.J.J., And R.J.A.P. PeEters (2010), "Homotopy Methods to Compute Equilibria in Game Theory," Economic Theory, 42, 119-156.

JaCKSOn, M.O., And A. WATts (2001), “The Existence of Pairwise Stable Networks," Seoul Journal of Economics, 14, 299-321.

JACKSON, M.O., AND A. Wolinsky (1996), "A Strategic Model of Social and Economic Networks," Journal of Economic Theory, 71, 44-74.

Jongen, H.Th., P. Jonker, And F. Twilt (1983), Nonlinear Optimization in $\mathbb{R}^{n}$, I. Morse Theory, Chebyshev Approximation, Methoden und Verfahren der Mathematischen Physik, 29, Peter Lang, Frankfurt.

LEMKe, C.E., AND J.T. Howson, JR. (1964), “Equilibrium Points of Bimatrix Games,” SIAM Journal on Applied Mathematics, 12, 413-423. 
Leung, M.P. (2020), “Equilibrium Computation in Discrete Network Games," Quantitative Economics, 11, 1325-1347.

LI, P., AND C. DANG (2020), “An Arbitrary Starting Tracing Procedure for Computing Subgame Perfect Equilibria," Journal of Optimization Theory and Applications, 186, 667687.

Mas-Colell, A. (1974), "A Note on a Theorem of F. Browder," Mathematical Programming, 6, 229-233.

Mas-Colell, A. (1985), The Theory of General Economic Equilibrium, A Differentiable Approach, Cambridge University Press, Cambridge.

MCLEnNAn, A. (2005), "The Expected Number of Nash Equilibria of a Normal Form Game," Econometrica, 73, 141-174.

MiYAuchi, Y. (2016), "Structural Estimation of Pairwise Stable Networks with Nonnegative Externality," Journal of Econometrics, 195, 224-235.

SCHANUEL, S.H., L.K. SimON, AND W.R. ZAME (1991), “The Algebraic Geometry of Games and the Tracing Procedure," in R. Selten (ed.), Game Equilibrium Models II: Methods, Morals and Markets, Springer-Verlag, Berlin, 9-43.

Stengel, B. von (1999), "New Maximal Numbers of Equilibria in Bimatrix Games," Discrete and Computational Geometry, 21, 557-568. 